\title{
ON A "MUCH UNDERESTIMATED" PAPER OF ALEXANDER
}

\author{
ALAN GLUCHOFF AND FREDERICK HARTMANN
}

\section{INTRODUCTION}

In 1915 there appeared in the Annals of Mathematics a paper entitled "Functions Which Map the Interior of the Unit Circle Upon Simple Regions," by J. W. Alexander II [2]. The author proposed to derive a number of sufficient conditions for a function $w=w(z)$ to map the interior of the unit disc in a one-to-one manner. These conditions could be "applied more readily" to $w(z)$ than the equivalent condition $\left(w\left(z_{1}\right)-w\left(z_{2}\right)\right) /\left(z_{1}-z_{2}\right) \neq 0$ for all $z_{1}$ and $z_{2}$ in the disc, which, as noted by Koebe, the reviewer of Alexander's paper in [64, p. 672], is "of little practical use." In doing so, Alexander developed several classes of univalent functions and some tests guaranteeing univalence which involve Taylor coefficients and location of zeroes and critical points, consequently opening up new areas of investigation for researchers in geometric function theory. Some prominent mathematical analysts, among them J. Dieudonné, G. Szegö, M. S. Robertson, R. Nevanlinna, L. Fejér, and L. Bieberbach picked up on topics introduced by Alexander, and work on them has continued throughout the century.

It is the purpose of this paper to analyze the contents of Alexander's work, detailing his highly intuitive arguments and how they were developed by later workers, and to follow several lines of development of this material. It is believed by some [5] that his paper was not appreciated for a long time after its publication, and the informal nature of its arguments might have led to its being described as "much underestimated" [19, p. 103]. Yet the work does introduce the notions of starlike functions, close-to-convex functions, functions of bounded turning, as well as other ideas and theorems which were rediscovered as much as twenty to forty years after its appearance, in some cases apparently without knowledge of Alexander's pioneering work.

Date: September 25, 2002.

1991 Mathematics Subject Classification. Primary: 30C45; Secondary: 30C50, 30C55.

Key words and phrases. Univalent functions, starlike, convex, close-to-convex. 
We will first give some biographical background on Alexander, then summarize briefly his mathematical contributions outside of the work under discussion. We will turn next to the paper itself, analyzing its contents section by section, and include several histories of themes initiated by Alexander, and how they developed in the modern era. Our history will concentrate most heavily on the era from 1915 to the late fifties and early sixties with an occasional reference to later work.

\section{Biography: James Waddell Alexander II (1888-1971)}

Alexander is probably much better known as a pioneering topologist in the first part of this century. This perhaps makes it all the more interesting that he should have made a contribution to the theory of univalent functions, though his arguments are clearly flavored with topological considerations.

Alexander's father, John White Alexander, was considered to be "one of America's great expatriate painters, his artistic accomplishments comparable to that of James McNiell, Whistler, Edwin Austin Abbey, and John Singer Sargent" [136, p. 43]. Among his larger works are murals in the Library of Congress and in the Carnegie Institute, the latter covering 3900 square feet of wall space with panels entitled "The Crowning of Labor." His later works include many studies of women holding, arranging, and enjoying flowers. He was awarded an honorary A. M. from Princeton in 1902. He died in 1915, the date of the appearance of his son's paper herein considered.

James Waddell Alexander II was born in 1888. As a child of two his parents brought him to Paris in a move that was intended to help his father recover from a severe attack of influenza. His father exhibited at the Société Nationale des BeauxArts and became friends with Whistler, Rodin, Henry James and Oscar Wilde, among others.

The son attended Princeton University, as had his great-grandfather (for whom he was named) and grandfather. He earned his B. S. in 1910, A. M. in 1911, and Ph.D. in 1915; in fact the paper under discussion was submitted as his doctoral dissertation. In 1917 he married Natalie Levitzkaya, whom he had met in Italy. He served in World War I in France, returning to America in July 1918. Working his way through the professional ranks at Princeton he achieved full professorship in 1928; in 1930 he was elected to the National Academy of Sciences. In 1933 he was appointed by Oswald Veblen to the staff of the School of Mathematics at the Institute for Advanced Study, where he remained until his retirement in 1951. 
Princeton, as well as the universities in Bologna and Paris, awarded him Honorary D. Sc. in 1947. Among the students with whom he came in contact during his years at Princeton were Morris Kline, as a research assistant, Albert Tucker, and Olga Taussky-Todd.

A favorite activity of Alexander and his wife was mountain climbing. In 1922 he made nine ascents of Long's Peak in Colorado, in 1924, ten ascents, having only visited the Rockies for the first time in 1921 for relief of allergies! A route along the east face of Long's Peak is now known as "Alexander's Chimney". He climbed peaks in the Alps as well, especially in the Chamonix area. Hassler Whitney tells a story of how Alexander and the topologist de Rham met: Alexander and his guide were crossing the rock tower at Chamonix when they and another group crossed paths near the top. Each group had left a pair of ice axes at the glacier, and decided to pick up the other group's axes when they reached the glacier again. When all parties met up at the lodging hut they found out they were already acquainted by name: Alexander and fellow topologist, De Rham [157, p. 108]. Alexander was also interested in the building of radio receivers; a design for a receiver circuit has been attributed to him.

After so active a life Alexander contracted polio in later years, and his health slowly deteriorated after his wife's death in 1967. He died on September 23, 1971, survived by a son and a daughter. He has been described as shy and retiring: a story by the well-known topologist S. MacLane [88, p. 217] relates that Alexander stepped out of the window of his first floor office at Princeton to avoid an unwelcome visitor! This shyness has resulted in little of a personal nature being known about Alexander.

\section{Mathematical Contributions}

We are not in a position to give a detailed account of Alexander's topological work here. The recent book, "History of Topology" [66] by I. M. James provides many references to his contributions, and further information as well as a complete bibliography can be found in [27]. Solomon Lefschetz has also left an informative memoir [80]. But we shall mention a few items here. As is clear from [88], Alexander, Veblen, and Lefschetz made some fundamental discoveries in the early years of the 20th century which made their home institution of Princeton a world leader in the new subject of algebraic topology. 


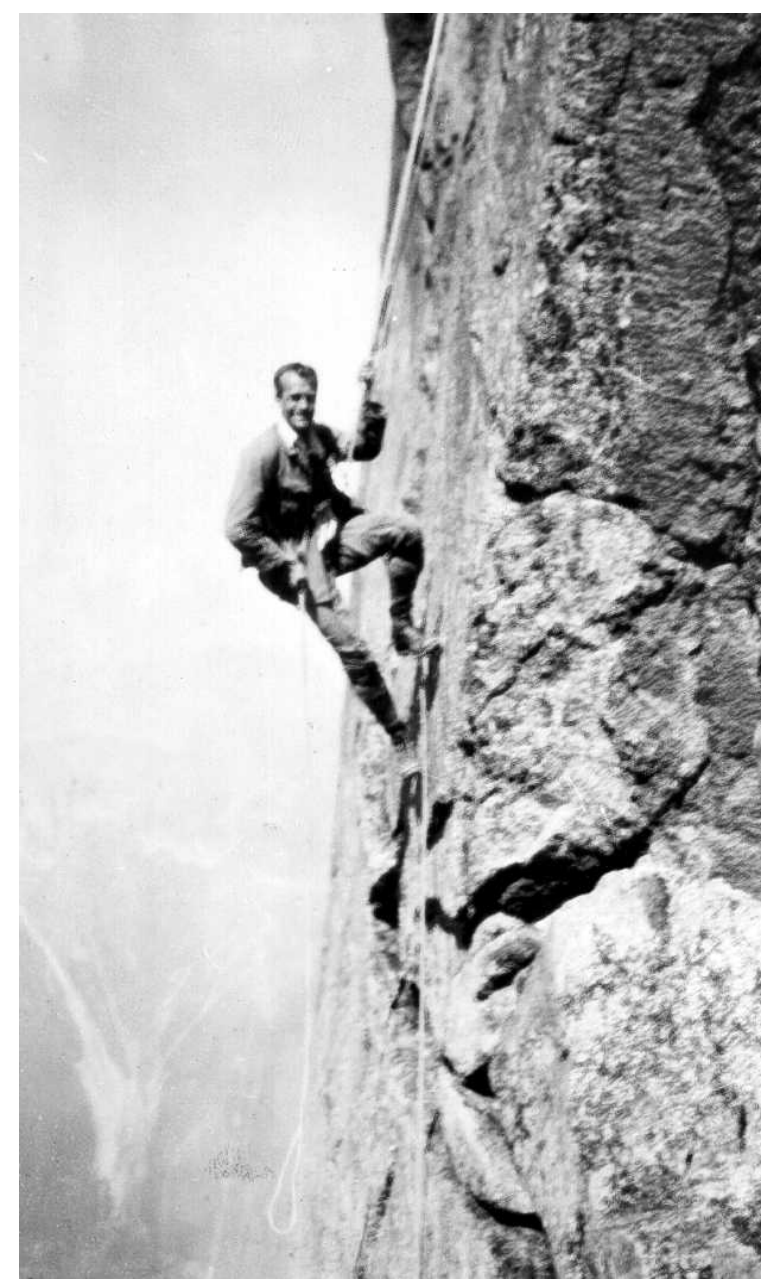

J. W. Alexander, II

(Princeton University Library)

Before our 1915 paper Alexander already had several papers in print, one coauthored by Veblen [27], in which he provided the fundamental work of Poincaré with "a reasonable element of logic" [66, p. 892]. In 1915 he proved the invariance of the Betti numbers and torsion coefficients of a manifold. (The Betti numbers and torsion coefficients were an early attempt to provide algebraic ways of distinguishing complexes.) In 1922 he established his Duality Principle: given a complex $\mathrm{K}$ embedded homeomorphically in an $n$-sphere, $S^{n}$, there is a precise relation between the homology groups of $K$ and $S^{n}-K$. In 1924 he produced what is perhaps his best known construct, the Alexander Horned Sphere: a simply connected surface $S$ (a 


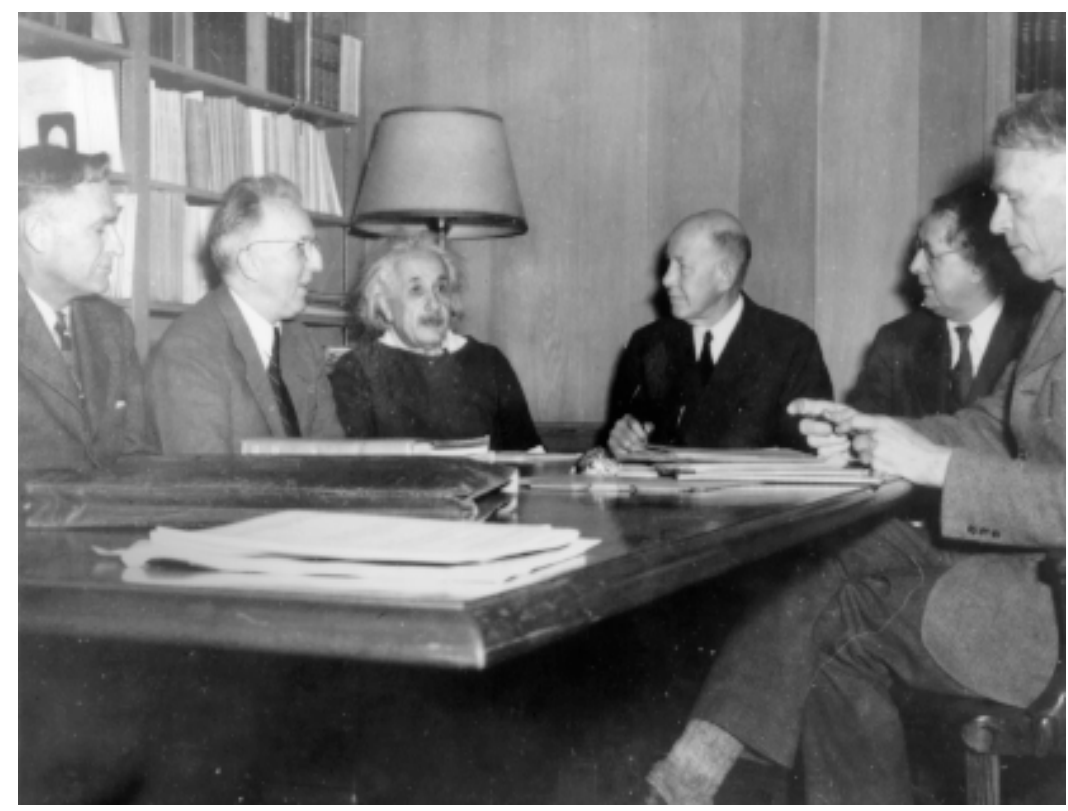

(L to R) J. Alexander, M. Morse, A. Einstein, F. Aydelotte, H. Weyl, O. Veblen at the Institute for Advanced Study (courtesy of the Archives of the Institute for Advanced Study)

homeomorphic image of $S^{2}$ ) in $S^{3}$, dividing $S^{3}$ into two regions, one of which is not simply connected. In 1928 came the discovery of the knot polynomial, an algebraic quantity which is an attempt to distinguish knots of different types. (The interest in knots and loops evidenced here recalls the types of arguments used in the univalent function paper.) It was in this year also that he received the Bôcher Memorial Prize for his paper, "Combinatorial Analysis Situs," in which combinatorial criteria for the homeomorphism of two complexes were given.

During his time at the Institute for Advanced Study Alexander wrote 14 papers on topics such as cohomology ring structure, and discrete Abelian groups and their character groups. In a paper of 1938 on "gratings" he attempted an elimination of points from topology entirely by defining a space to be an element of a Boolean algebra in which there is defined a class, $O$, of elements which are the open sets. Such works among others have elicited the opinion that Alexander was the "leading American topologist of his period." [65] 


\section{The Univalent Mapping Paper}

Univalent function theory is generally regarded as having begun in 1907 with a publication by P. Koebe [77] (the reviewer of Alexander's paper mentioned in the Introduction) in which he proved that there is an absolute constant $k$ such that the disk $|w|<k$ is contained in the image of every one-to-one mapping $f(z)=z+a_{2} z^{2}+$ $a_{3} z^{3}+\cdots$ of the unit disc, and also proved the existence of bounds on $\left|f^{\prime}(z)\right|$ which depend only on $|z|$. (Without loss of generality one may choose $f(0)=0$, since this is a mere translation of the image, and $f^{\prime}(0)=1$, since $f$ is one-to-one on the unit disk, hence $f^{\prime}(0) \neq 0$ and therefore may be chosen to be 1.) Efforts of mathematicians then focused on providing exact bounds for these and other constants, for example, those related to the growth of $|f(z)|$ itself. The theorems involved finding bounds on the coefficients of power series expansions of auxiliary univalent functions and relating these estimates to the functions themselves. These results can be found, for example, in [32] and [130], and comprise what is now, eighty or so years later, known as "elementary univalent function theory." Thus the focus early on was on problems related to the entire class of univalent, or schlicht, functions. Another stream feeding the work was research related to the Riemann Mapping Theorem and conformal mapping in general; in particular boundary behavior of conformal maps attracted much attention in these years.

An outstanding figure in the early development of geometric function theory was Ludwig Bieberbach (1886-1982). His doctoral dissertation, completed in 1910, was on automorphic functions. He taught at Zürich, Königsberg, Frankfurt, and finally succeeded C. Carathéodory at Berlin University in 1921. He was a prolific writer of textbooks, producing works on numerical analysis, calculus, conformal mapping, and complex function theory. In 1916 he conjectured his well-known bound for the coefficients of a normalized univalent function $\left(\left|a_{n}\right| \leq n\right)$ and proved the bound for the case $n=2$; the conjecture was not proved in general until 1984. Bieberbach produced many papers on univalent function theory in the early 1920's.

An important precursor to Alexander's paper in its consideration of specific classes of univalent mappings was [137], in which E. Study considered among other things the class of convex mappings, that is, analytic mappings defined on the unit disc of the complex plane which map in a one-to-one way onto a convex domain. He proved that such mappings also send each disc centered at the origin having radius 
smaller than one to a convex domain as well, and provided what became a wellknown analytic formulation that a univalent map be convex on the unit disc: this occurs if $1+\Re\left\{z f^{\prime \prime}(z) / f^{\prime}(z)\right\}>0$ there, provided $f^{\prime}(z) \neq 0$ on the disc.

Into this setting came Alexander's paper, at a time before univalent function theory became divided into well-defined areas dealing with function classes, extremal problems, convolution operators and the like. His stated goal was, as noted above, to provide useful sufficient conditions that a given mapping be univalent. In his work he provided conditions on the zeroes, critical points, derivative, and on Taylor coefficients of an analytic function which ensure univalence. This is a commonplace approach now; there are many modern papers which state conditions on these quantities and deduce a special consequence from them which implies univalence, but Alexander's was one of the first to do this. An additional feature of the work is that the consequence of univalence always follows from implications that the boundary of the unit circle or a segment joining two points interior to the unit disc is mapped onto a non-self-intersecting curve. The arguments which support these conclusions are mostly geometrical. As such they stand in contrast to many of the papers of the day and now, where one is used to much more manipulation of analytical formulae like Study's to achieve the desired end. In his efforts to have the boundary image avoid self-intersection Alexander introduced conditions such as starlikeness, which have become the definitions of now thoroughly investigated subclasses of the monolithic schlicht class. Another characteristic of the paper is its focus on polynomials; every function class defined is motivated by the desire to produce examples of schlicht polynomials.

The paper occupies a position in the intersection of three mathematical areas: the theory of polynomials and their roots and critical points (considered a subfield of algebra at the time), univalent function theory, and the topology of boundary curves of differentiable mappings. Each of these areas will be seen to play a role in Alexander's methods and those of his successors. It appears that the paper had somewhat of a belated reception; there are relatively few references to it in the remaining years of the teens and even the 1920's. It is only in the late 1920's and the early 1930's that it came into some prominence, perhaps due to the publication of Montel's and Bieberbach's books on univalent function theory [14], [95].

An indication of the eventual importance attached to the paper was its inclusion in the bibliography of J. Dieudonné's 1938 survey [31] of the analytic theory of 
polynomials. Jean Dieudonné (1906-1992) was a leading mathematician of the $20^{\text {th }}$ century and a founder member of Bourbaki, whose interests ranged from point-set topology to linear functional analysis, classical groups over general fields, and Lie groups. The Bourbaki books formed a series of volumes on mathematical topics which attempted to standardize the presentation of material from disparate fields. In view of this degree of interest in abstract mathematics it is interesting to note that the first eight or so years of Dieudonné's mathematical career, beginning with his thesis in 1931, were devoted to research on complex polynomials. We shall detail some of his work stemming from Alexander's paper below.

\section{Zeroes In A Half-Plane and Univalent Polynomials}

Alexander began by dealing with polynomials. He first described a polynomial of degree $n$ as a conformal mapping of the plane onto an $n$-sheeted Riemann surface, with the exception of the branch points of the image. (Arguments involving Riemann surfaces never explicitly enter into the paper, but one is reminded, in his dealings with the loops and curves in the image space, of the corresponding manipulations of curves on Riemann surfaces which give rise, for example, to permutation groups of the roots of polynomials.) His first observation was that it is evident from the form $w=\left(z-z_{1}\right)\left(z-z_{2}\right) \ldots\left(z-z_{n}\right)$ that "the change in the argument of $w$ as $z$ varies from $z^{\prime}$ to $z^{\prime \prime}$ is equal to the sum of the angles generated at the roots of $w$ by the vectors joining the latter to $z$..." (This phrasing shows Alexander's penchant for long descriptive sentences rather than equations.) Thus if the $z_{k}$ all lie on the same side of a straight line, then the line is mapped to a curve of monotone argument ("continuously turning about the origin"), and if the critical points are all on the same side of a line, the line is mapped into a curve with monotone tangent vector argument ("curvature is either everywhere positive or negative"). These facts follow from the interpretation of $\left(z-z_{k}\right)$ as the vector from $z_{k}$ to $z$.

His specific goal in $\S 1$ through $\S 3$ was to determine a maximal region, $R_{n}$, such that every polynomial of degree $n$ whose roots are in the region is univalent ${ }^{1}$. By consideration of the mapping $w=(a-z)^{n}$ he pointed out that $R_{n}$ can contain no point closer than $\csc (\pi / n)$ to the origin, or else the above map with $a=\csc (\pi / n)$ will stretch the unit disc around to achieve self-intersection. He then completed the region by drawing a vertical tangent, $T$, to the unit circle through the point $z=1$,

\footnotetext{
${ }^{1}$ Section numbers refer to the sections of Alexander's paper. §1: p. 12, §2: pp. 12-13, §3: pp. 13-15, §4: pp. 15-16, §5: pp. 16-18, §6: p. 18, §7: pp. 19-20, §8: pp. 20-22, §9: p. 22
} 
and took as $R_{n}$ the set of points to the right of the tangent line and outside of the circle $|z|=\csc (\pi / n)$.

Alexander argued as follows: by the well-known Gauss-Lucas Theorem [90, p. 22 ], if the roots of $w$ are in $R_{n}$, then the critical points of $w$ lie to the right of $T$ as well. Thus, as noted before, any line segment to the left of $T$ will get mapped to a curve with argument and tangent vector argument simultaneously either increasing or decreasing. "As a consequence of this, two points $z^{\prime}$ and $z^{\prime \prime}$ of the left half plane $[\Re(z)<1]$ correspond to the same point of $w$ only if the linear segment $z^{\prime} z^{\prime \prime}$ is mapped upon a curve which encloses the origin. But this is impossible when $z^{\prime}$ and $z^{\prime \prime}$ are within or on the unit circle, for they then subtend an angle of less than $2 \pi / n$ at each of the roots of $w$, or in all an angle less than $2 \pi . "$ A folding of the image of such a line segment back on itself or formation of loops is ruled out by the constant sign of the curvature and tangent vector argument change, though this point was not explicitly raised by Alexander. Thus the region is established. Maximality of the region was demonstrated by showing that, given any point $p$ outside the region, one can construct a non-univalent polynomial having $p$ as a zero.

Let us observe that the argument as stated has a limitation: clearly, if $z^{\prime}$ and $z^{\prime \prime}$ are two points in the left plane corresponding to the same point of $w$, the line segment connecting them, when extended in both directions, need not have all the roots and critical points of $w$ to the right of it; only a vertical line necessarily satisfies this condition. Thus Alexander's argument would establish the univalence of the polynomial on vertical line segments of the unit circle, from which overall univalence would then have to be deduced. Perhaps this was in his judgment an obvious deduction.

This result has been quoted by later writers; [141, p. 31] is a recent citation. It is interesting to note a parallel line of development which drew the attention of several prominent complex analysts: Hurwitz's stability criteria. Alexander's condition above implies that the zeroes are in $\Re(z)>1$, and restricting the zeroes to the half-plane $\Re(z)>\csc (\pi / n)$ ensures that they satisfy Alexander's condition, thus the restriction of the zeroes of a polynomial to a right or left half plane is a relevant consideration. This condition on the zeroes reminds one of Hurwitz polynomials, which by definition have all their zeroes in the left half plane. These polynomials arose first in connection with the study of stability of linear systems, a study going back to James Clerk Maxwell's 1868 paper "On Governors" [91]. In this work he 
examined the differential equations describing control systems and pointed out that for stability of the system all the characteristic roots of the auxiliary polynomial must have negative real part. He set the problem of determining the conditions on the coefficients of the polynomial (obviously the given quantities in a physical system) in order that the zeroes lie in the left half-plane. (See [9], [44] for more detailed accounts of the history of this problem). Several workers provided partial or complete answers in the ensuing years, including A. Hurwitz.

Adolf Hurwitz (1859-1919) was a mathematician with a wide range of interests in algebra, number theory, Fourier series and complex analysis. His Ph.D. thesis on modular functions, awarded in 1881, was supervised by Felix Klein at Leipzig. Hurwitz held positions at Königsberg and later at Eidgenössische Polytechnikum Zürich, having taught and become a close friend of Hilbert during these years. Some of his contributions to complex function theory play a role in the story of the reception of Alexander's paper.

In 1895, Hurwitz published a paper [60] in which he provided a necessary and sufficient condition that a polynomial with real coefficients have zeroes in the left half-plane, the condition being stated in terms of matrices of the coefficients. It is interesting that Hurwitz was moved to this investigation "at the suggestion of my esteemed colleague A. Stodola", an engineer who used the results of the paper in theoretical work and practical application on the turbines of the Davos Spa Turbine Plant. In honor of this paper, polynomials with real coefficients having zeroes in $\Re(z)<0$ are now called Hurwitz polynomials. Research on Hurwitz polynomials continued in Alexander's day; a 1914 paper reduced the number of determinant calculations in Hurwitz's criteria by half [82], and Nikola Obreschkoff, whose work on starlike polynomials will be mentioned in connection with later sections of Alexander's paper, gave in 1939 a simple proof of the Hurwitz criteria [102]. Alexander's paper is the only reference the authors have discovered which mentions the univalence properties of Hurwitz-like polynomials.

We conclude this section by noting that in a sense Alexander can be seen as having attempted his first definition of a geometric sub-class of univalent functions in these sections, since he was essentially examining the class of mappings, $w$, analytic on the unit disc for which $d(\arg (w)) / d y<0$ and $d(\arg ($ tangent vector at $w)) / d y<0$ at each point of the disc, with total change in $\arg (w)$ on any vertical line traversed upward greater than $-2 \pi$. Translating such a local geometric condition into global 
univalence is a theme of his paper, indeed one of its innovations, though he fell short of a rigorous treatment in several cases. The class defined here is of the same type as the starlike, convex, bounded turning and vertically convex classes to be considered later. For these the translation was successfully done with rigor by later mathematicians.

We also have here the first example in the paper of the mix of material related to the location of polynomial roots and results from analytic function theory, an intersection which puts him in the company of certain mathematicians in the first half of the twentieth century. G. Szegö, N. Obreschkoff, A. Hurwitz and S. Kakeya felt equally at home in polynomial theory and univalent function theory. A 1929 survey by VanVleck [151] details more material in this intersection.

\section{Starlike And Convex Polynomials}

Section 4 of Alexander's paper deals with convex and starlike mappings. He defined a star-shaped region as one "every point of which may be joined to a point ' $a$ ' by means of a linear segment consisting only of points in the region"; the point $a$ is called the center. "When an arbitrary point of the region may be chosen as the center, the region will be called convex." He introduced the idea of a mapping $w$ of the unit disc onto a starlike or convex region in an attempt to ensure univalence of the mapping by controlling the shape of the boundary image: by insisting that it be starlike or convex, overlapping or looping is avoided and hence univalence is achieved. (This objective is never explicitly stated, but it seems clear that this was his goal in introducing these classes.) The two types of mappings are characterized geometrically: a mapping $w=w(z)$ is starshaped if $\arg (w)$ is a never-decreasing function of $\theta=\arg (z)$ as $z$ describes the unit circle in the counterclockwise direction, and it is a convex function if the argument of the normal vector, $z(d w / d z)$, of the image curve is a non-decreasing function of increasing $\theta$. Specific examples of starshaped mappings had, of course, been available since the Schwarz-Christoffel studies, and Study even included in his work an example of the integral formula for "... a 10-gon, which corresponds to the figure of the Pentagramma Mysticum" [137, p. 77]. But whereas convexity in general was a well-established idea by this time, starlikeness was somewhat novel.

Before considering the theorems in this section we should register some difficulties. Clearly a mapping $w$ may have a "star-shaped" or convex boundary image and not be univalent: $w=z^{2}$ is an easy example. Furthermore, a mapping may map the 


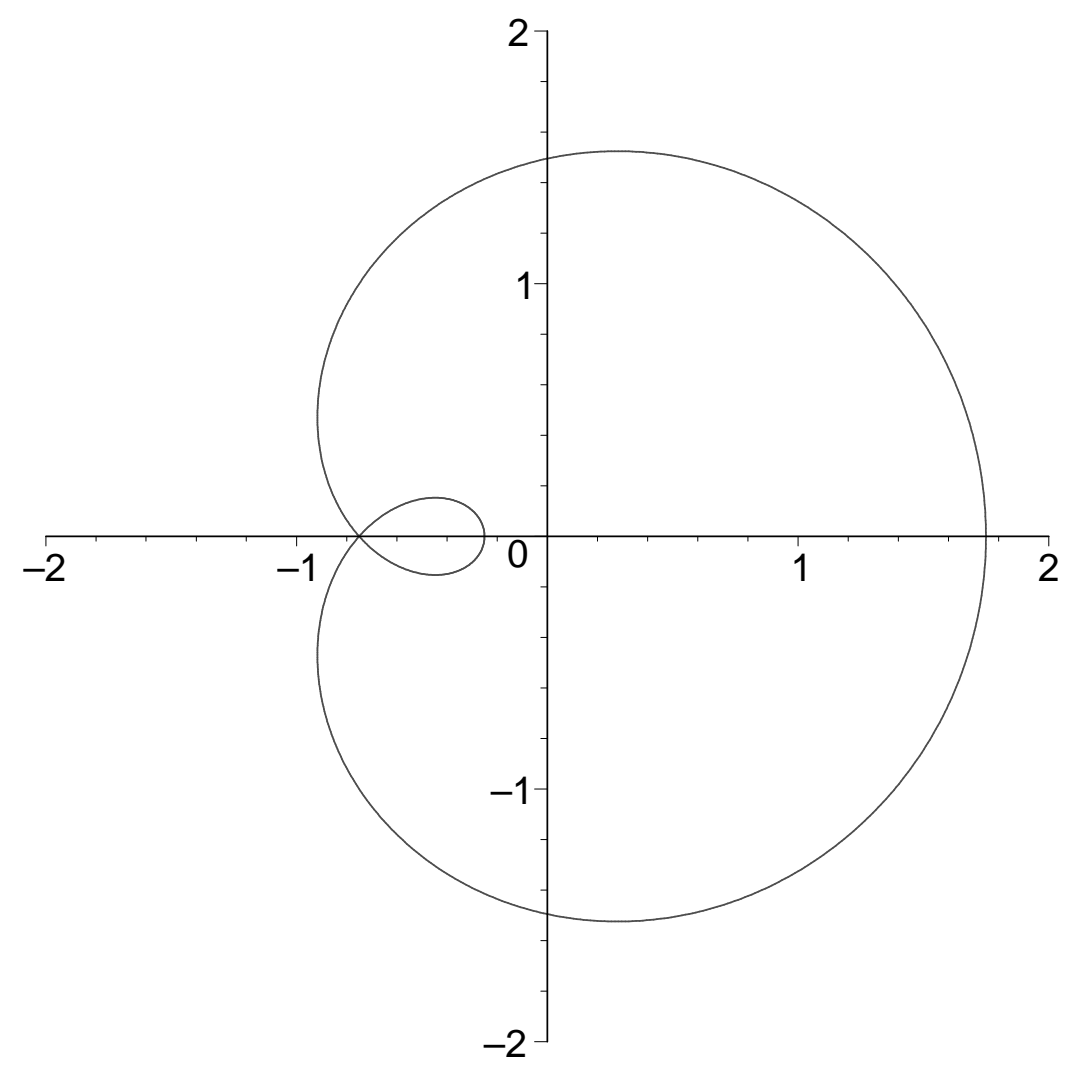

Figure 1: $f(z)=z+\frac{3}{4} z^{2}$

interior of the unit disc onto a starshaped region and have its boundary curve fail the condition $(d / d \theta)(\arg w(\theta))>0$ at some points: $w=z+(3 / 4) z^{2}$ is an example which also fails univalence, see Figure 1. (All figures are provided by the authors, as Alexander supplied none in his paper.) Finally, the univalence of a mapping in the interior of the unit circle does follow from univalence on the boundary, but Alexander never explicitly stated this, apparently assuming it obvious. It seems that, as in the previous section, he was unable to make the transition from a local condition for univalence (increasing argument of the image) to global univalence. This transition was made rigorously in the late 1920's and early 1930's by employing Study's condition for convexity and the related 1921 condition of Nevanlinna [98] for starlikeness: $\Re\left[z f^{\prime}(z) / f(z)\right]>0$ for $|z|<1$. The results can be found, for example, in Montel's 1933 volume [95, p. 13] and Bieberbach's 1930 two-volume treatise [14, V. 2, p. 93]. These approaches have become the standard modern treatment of this material. 
There are two main theorems in $\S 4$, we will treat the second one first. Again, Alexander considered polynomials. The goal here is similar to that of the previous section: find a maximal region $S_{n}$ such that any polynomial $z P_{n-1}(z)$ having the $n-1$ roots other than zero in $S_{n}$ will map onto a starlike region. By first considering $w=z(z-a)^{n-1}$ with $d w / d z=(z-a)^{n-2}(n z-a)$ he pointed out that the region must omit $|z|<n$. The region $|z| \geq n$ is actually the desired region: if the remaining zeroes $z_{k}, k=1 \ldots n-1$ have $\left|z_{k}\right| \geq n$, then the angle each makes with an element $d$ s on the unit circle is less than $d s /(n-1)$. Thus from $\arg (w)=\arg (z)+\Sigma \arg \left(z-z_{k}\right)$ it follows that $d / d s(\arg (w))>d s-\Sigma\left|d / d s\left(\arg \left(z-z_{k}\right)\right)\right|>0$, and we have local positive argument change on the image curve. (Alexander did not argue using these inequalities, but rather provided a verbal description of this situation.) Thus the boundary curve is star-shaped. The maximality follows from the example $z(z-$ $n)^{n-1}$.

Alexander's proof can be expressed in purely analytical terms, as was done for example by T. Başgöze in 1969 [7]. Başgöze argued that if $P_{n-1}(z)$ has all its zeroes, $z_{1}, \ldots, z_{n-1}$ outside the unit disc, then for $|z|=r,\left|z_{k}\right|=r_{k}, f(z)=z P_{n-1}(z)$ we have $\Re\left\{z f^{\prime}(z) / f(z)\right\}=1+\Re\left\{\Sigma z /\left(z-z_{k}\right)\right\} \geq 1-\Sigma r /\left(r_{k}-r\right) \geq 1-(n-1) r /(1-r) \geq$ 0 if $r<1 / n$, an equivalent form of Alexander's result. But it is clear that the kernel of this proof was contained already in Alexander's argument, which could be restated as: $\arg \left(z P_{n-1}(z)\right)=\arg (z)+\sum \arg \left(z-z_{k}\right)$ implies $d\left(\arg \left(z P_{n-1}(z)\right)\right)=d \theta+$ $\Sigma d\left(\arg \left(z-z_{k}\right)\right) \geq d \theta-\Sigma(r d \theta) /\left(\left|z_{k}\right|-r\right) \geq d \theta-\Sigma r d \theta /(1-r)=d \theta[1-(n-1) r /(1-r)]$, thus dovetailing with Başgöze's calculation. Alexander did not take the route of introducing $d\left(\arg \left(z-z_{k}\right)\right) / d z=\Re\left(z /\left(z-z_{k}\right)\right)$, but just dealt directly with the differential angle change. In spite of the lack of rigor in Alexander's approach, it does give a direct feel for the role of pushing the zeroes of the polynomial away from the origin to ensure a simple boundary curve image.

An early strengthening of this result was given in a note by N. Obreschkoff in 1934 [101] which begins as Alexander did by setting $f(z)=z+a_{2} z^{2}+\cdots+a_{n} z^{n}$ and $\Phi=\arg f=\theta+\sum_{k=1}^{n-1} \arg \left(z-z_{k}\right)$ for $z=e^{i \theta}$, noting $(d / d \theta)\left(z-z_{k}\right)>-r /\left(r_{k}-r\right)$ for $r<r_{k}$, and getting $d \Phi / d \theta \geq 1-\sum_{k=1}^{n} r /\left(r_{k}-r\right)=\left(r f_{0}^{\prime}(r)\right) / f_{0}(r)$ where $f_{0}(z)=z\left(z-\left|z_{1}\right|\right) \ldots\left(z-\left|z_{n-1}\right|\right)$. It is then noted that $\left(r f_{0}^{\prime}(r)\right) / f_{0}(r) \geq 0$ if $r<a$, where $a$ is the closest critical point of $f_{0}$ to the origin. Thus $f$ is starlike on $|z|<a$. Now if the $\left|z_{k}\right| \geq n$, it can easily be seen that $a \geq 1$, so "One obtains easily as a 
particular case a theorem of Alexander" [p. 2050]. (It is interesting that this note occurs under the heading "Algebra" in Comptes Rendus.)

This theorem of Alexander's as well as the conjecture considered in $\S 5$ were the subject of extended works by Dieudonné [30] and Szegö [145], each of whom acknowledged the pioneering work discussed in these sections. In addition, it was included in Montel's 1933 book [95], where it was deduced as a corollary of theorems related to Szegö's earlier work on convolution results for polynomials. Alexander's work was cited, and a generalization due to Biernacki [15] in 1927 to multivalent polynomials was given. P. Montel, the advisor of both Dieudonné's and Biernacki's theses, had introduced the notion of a $p$-valent function: "A function is said to be $p$-valent in a domain, $D$, if it takes no value more than $p$ times in this domain and there exist values which it takes exactly $p$ times" $[15$, p. 622]. In his thesis Biernacki said (p. 628) that Montel suggested to him that he attempt to generalize Alexander's result to $p$-valent polynomials. This he succeeded in doing by producing the following result: if a polynomial of degree $n$ vanishes $p$ times at the origin and nowhere else in the unit circle, then the polynomial is necessarily $p$-valent in the circle $|z|<p / n$. The functions $f(z)=z^{p}(z-a)^{n-p}$ with $|a|=1$ are extremal.

Alexander's other theorem in $\S 4$ is a connection between convex and starlike mappings: "A necessary and sufficient condition that the function $w$ map the interior of the unit circle upon a convex region is that $z(d w / d z)$ shall map the interior of the unit circle upon a star-shaped region with center at the origin." His argument is again geometric: $w$ maps the unit circle onto a convex region if and only if the argument of the normal vector $z(d w / d z)$ to the region is an increasing function of $\theta=\arg (z)$ as $z$ traverses the unit circle in the counterclockwise direction. But this latter condition holds if and only if $z(d w / d z)$, itself regarded as a mapping, maps upon a star-shaped region. This theorem together with the first considered allows a corollary: a polynomial $w$ of degree $n$ maps the interior of the unit circle onto a convex region if the critical points $w_{k}$ all satisfy $\left|w_{k}\right| \geq n$. This region is maximal.

Unfortunately, the difficulties mentioned before still dog us here; without some further restriction on hypothesis, the theorem is false: $w=z^{2}+(3 / 4) z^{4}$ is a mapping with star-shaped image (though not univalent) see Figure 1 , yet $z(d w / d z)=2 z^{2}+$ $3 z^{4}$ is not convex (and also not univalent). Modern treatments clarify the situation by pointing out that the precise statements of the analytic forms of these conditions noted above allow the immediate conclusion that $f(z)=z+a_{2} z^{2}+\cdots$ is univalent 
and convex if and only if $z f^{\prime}(z)$ is univalent and convex. In this form the result is usually given the name Alexander's Theorem, e.g. in [32, p. 43]. But again the intuition provided by Alexander's argument is worth pondering, since the modern treatment, with its direct substitution of functions into conditions, can easily mask the geometry.

This argument was repeated in 1917 in a paper by C. Loewner [83] in which he considered bounds on the quantity $\left[d w /\left.d z\right|_{z=z_{1}}\right] /\left[d w /\left.d z\right|_{z=z_{2}}\right]$ for $\left|z_{1}\right|,\left|z_{2}\right|<\rho$; he noted only that if $f$ is convex, then $z f^{\prime}(z)$ is univalent. He failed to mention the starlikeness of $z f^{\prime}(z)$, and was apparently unaware of Alexander's work.

How did the idea of starlike mappings come about? The notion of a star-domain seems to have originated in a series of five notes by G. Mittag-Leffler on analytic continuation published from 1900 to 1905 [94]. In these notes he attempted to find a maximal domain of analytic continuation for a power series and succeeded in deriving a multiple series which converges in a domain starlike with respect to the center of the series. The novelty of the star domain idea is expressed: "Pour traiter à fond la question que nous avons posée, il faut définir un domain $\mathrm{K}$ qui soit aussi vaste que possible. C'est ce que nous allons faire en introduisant une nouvelle conception géométrique: l'Étoile" [I, p. 47]. The star domain seems to have been closely associated with Mittag-Leffler; Bieberbach in a 1919 paper [11, p. 303], for instance, said "by a star with center $z=0$ we understand with Mittag-Leffler a domain...", another author referred to "une étoile de Mittag-Leffler" [59, p. 101] in a discussion of cells of univalence for polynomial mappings.

There may have been a more direct source giving Alexander the idea for the shape. At the end of his paper [§9] Alexander said, "The author is happy to express to Professor T. H. Gronwall his indebtedness for much useful advice during the preparation of this paper." Thomas Hakon Gronwall was instructor at Princeton in 1913-1914 and assistant professor there during 1914-1915, and he had done some work in univalent functions: his 1914 area theorem is the standard starting point for the modern treatment of "elementary" univalent function theory. In [53] he established bounds for the growth of $|f(z)|$ and $\left|f^{\prime}(z)\right|$ for one-to-one mappings of $|z|<1$ onto a bounded domain, and in two notes [54], [55] he stated similar growth bounds for general univalent mappings of the disc, as well as general convex mappings. In [55] the growth rates were determined for mappings onto a domain which "reproduces itself by a rotation of $2 \pi / n$ about the origin"; such domains 
call to mind starlike images, though they need not be starlike. In an interesting coincidence, Gronwall was an assistant professor in Stockholm during the 1897-1898 academic year, the latter year being the same in which Mittag-Leffler presented his results to the Academy of Sciences in Stockholm and in which they originally appeared in print. It seems clear that Gronwall's presence influenced Alexander's interest in geometric function theory.

Treatment of the starlike class continued in a 1921 paper by Nevanlinna [98] mentioned earlier. Rolf Nevanlinna (1895-1980) was born in Finland to a family many of whose members showed a talent for mathematics. He studied at the University of Helsinki, largely under the guidance of Ernst Lindelöf. He accepted positions at the University of Zürich, Göttingen, and Helsinki, though his regular professorship was at Helsinki. His greatest contribution to mathematics was his value distribution theory in complex variables, developed in part with his brother Frithiof, a theory which now goes under the name Nevanlinna Theory. The 1921 paper [98] was published soon after his thesis, which was defended in 1919. As noted, the paper was the first to contain the analytic condition $\Re\left(z f^{\prime} / f\right)>0$ for starlikeness, and the "Bieberbach" bound $\left|a_{n}\right| \leq n$ was proved for the starlike class. This latter theorem was the first attempt to prove the bound for subclasses of the univalent class, an activity which continued throughout the development of geometric function theory: the creation of new function classes presented new opportunities for the verification of the bound for more univalent functions. Nevanlinna cited Bieberbach's 1919 paper [11], repeating its use of "a star of Mittag-Leffler" terminology, and cited Alexander's relation between the starlike and convex functions.

Nevanlinna's proof of the analytic condition for starlikeness is a careful piece of analysis in which he used approximations of the starlike domain by domains $S_{n}$ consisting of polar coordinate arcs and segments and established the condition $\frac{d}{d t} \arg \left(f_{n}\left(e^{i t}\right)\right)=\Re\left(z \frac{d}{d z} \log \left(f_{n}(z)\right)\right)>0$ for each approximating conformal map $f_{n}: S_{n} \rightarrow \mathbb{C}$. Corners of $S_{n}$ were treated separately, the Poisson integral formula was invoked to move the condition to the interior of the unit disc, and a limit was taken to pass the condition to the original mapping function $f$. In short his treatment was a rigorous analytic proof of the equivalence of $f$ univalent starlike and $\Re\left(z \frac{d}{d z}\left(\log \left(f_{n}(z)\right)\right)>0\right.$ on the interior of the unit disc.

Generalizations of the class appeared in the 1930's. In 1932 L. Spaček [133] defined the class of spiral-like functions and in 1936 M. Robertson [120] introduced 
the classes of functions starlike of order $\alpha$. (Malcolm S. Robertson, a prominent Canadian univalent function theorist, who has done work in function classes, convolution theory, variational formulae, and subordination techniques beginning in 1932, contributed much to the early development of geometrically defined function classes.)

As for Alexander's Theorem ( $f$ convex if and only if $z f^{\prime}$ starlike), which is probably the best known result of the paper, it found its way into many early papers. In [12] Bieberbach showed how sharp growth bounds for $|f(z)|$, where $f$ is convex, can be obtained from bounds on $\left|z f^{\prime}(z)\right|$, where $z f^{\prime}$ is starlike, using integration, and in [11] he obtained the rotation bound $\left|\arg f^{\prime}(z)\right|<2 \arcsin |z|$ for convex $f$, and from Alexander's Theorem obtained $\left|\arg \left(w^{\prime}\right)\right| \leq 2 \arcsin |z|+\pi / 2$ for $w$ starlike. (Incidently, he used the curiously dismissive phrase "die natürlich leicht beweisbare Bemerkung" [11, p. 303] to describe Alexander's result.) These were just some early instances of the ease with which results about one class could be used to derive results about the other by using the theorem, a technique which has been used many times since.

The idea of moving from one class of univalent functions to another via the mapping $f \rightarrow z f^{\prime}(z)$ or its inverse $f \rightarrow \int(f(t) / t) d t$ was soon applied to other sets of functions. Robertson derived one such relation. Fejér in 1933 had introduced the class of vertically convex functions: these are analytic functions $f=u+i v$ on the unit disc with real coefficients satisfying $d / d \theta\left(u\left(r e^{i \theta}\right)\right)<0$ for all $r<1$. Such functions are univalent. In $1932 \mathrm{~W}$. W. Rogosinski introduced the class of typically real functions: they are functions $f$ analytic on $|z|<1$ with real coefficients satisfying $\Im(z) \Im(f(z)) \geq 0$ in the disc; these need not be univalent, but Robertson in $[120$, p. 392] observed that by applying the Alexander integral operator to such an $f$ "the loops will be removed" and a vertically convex function results. The search for such relations among classes has come to be known as looking for "Alexander-type" transforms, and his particular differentiation-integration transform has been called "the first integral operator defined on a subclass of the schlicht class" [93, p. 148].

Alexander's work on polynomials motivated a question in the more general setting of univalent functions. The polynomial theorem in $\S 4$ can be considered in the following way. Let $C_{n}$ be the class of polynomials $P(z)=z+a_{2} z^{2}+\cdots+a_{n} z^{n}$ of degree $n$ having zeroes, $z_{k}, k=1 \ldots n-1, z_{k} \neq 0$ satisfying $\left|z_{k}\right| \geq 1$. Then Alexander's result shows that the radius of univalence and radius of starlikeness 
for the class are both equal to $1 / n$, which is also the greatest lower bound of all critical points of functions in the class (recall the example $w=z(z-(1 / n))^{n-1}$ ). In Dieudonné's thesis [29] he considered the class $E_{M}$ of functions $f(z)=z+$ $a_{2} z^{2}+\cdots$ analytic in the unit disc for which $|f(z)| \leq M$, and found that the radius of univalence over this class is $M-\sqrt{M^{2}-1}$, with the extremal functions being $f(z)=M z\left(e^{i \theta}-M z\right) /\left(M e^{i \theta}-z\right)$. He then proved (p. 350) that these extremal functions map the unit disc onto a starshaped domain. In a footnote on this page he remarked that his result is the third he had encountered in which a family of functions has the radius of univalence, radius of starlikeness, and minimum modulus of critical points in the family all coinciding, the first two instances being $\S 4$ and $\S 6$ of Alexander's paper. He was then led to say: "It would be interesting to research whether it is a question of simple coincidence, or if, in general, when two of these limits are equal, the third is equal to them." We shall return to this question in a later section.

\section{Alexander's Conjecture, Kakeya's Theorem}

Having established in the preceding section that the position of the critical points of a polynomial $w$ of degree $n$ can determine whether the image of the map is convex, hence "nonoverlapping", it was natural for Alexander to ask the same sort of maximal region question as was considered before: find the greatest region in which the critical points of $w$ can lie in order that $w$ be univalent. Alexander did not answer this question completely, but gave a partial result and the correct conjecture in $\S 5$.

He began by noting that the region must lie in $|z|>\csc (\pi / n)$, as his example $(z-\csc (\pi / n))^{n}$ shows. He then determined another circle of larger radius centered at the origin whose exterior is contained in the desired maximal region. Again his arguments are geometric, and his goal was to prevent the image of the unit circle $C$ from overlapping itself. He wanted to prevent either a positive loop or a negative loop from appearing in the image of $C$, and to this end it is clear that the critical points must be driven from the interior of the unit disc. This action, according to Alexander, prevents any positive looping in $C$; now negative loops must be dealt with.

A look at the series of images under $w=(z-1.1)^{3}$ of concentric circles centered at the origin with decreasing radii, $r$, shown in Figure 2 shows how a negative loop can indeed occur in the unit disc even if the critical points lie outside $C$, the 


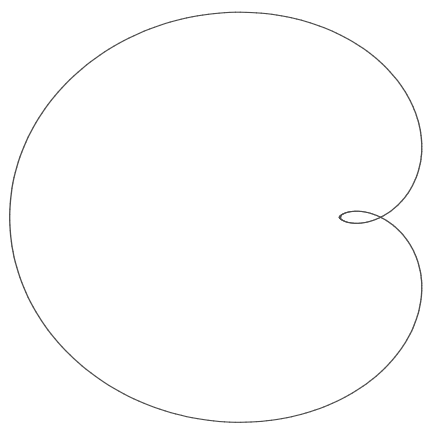

Full map $r=1.10$

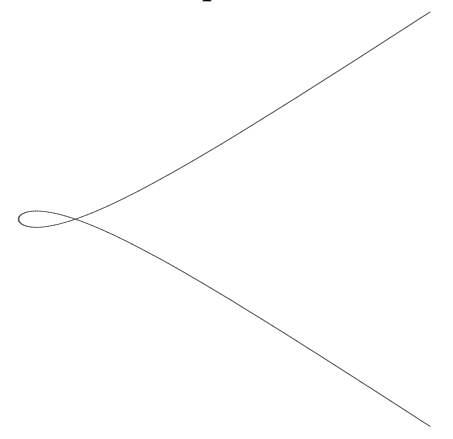

$$
r=1.05
$$
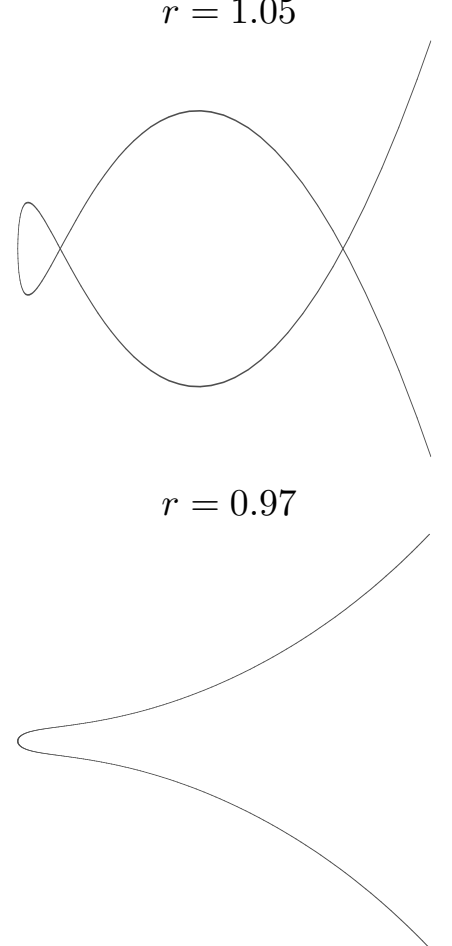

Corner detail, $r=0.90$

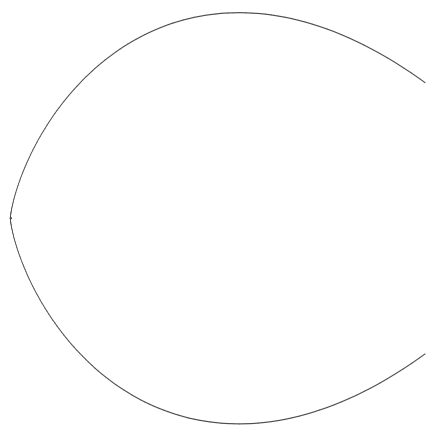

Loop detail, $r=1.10$

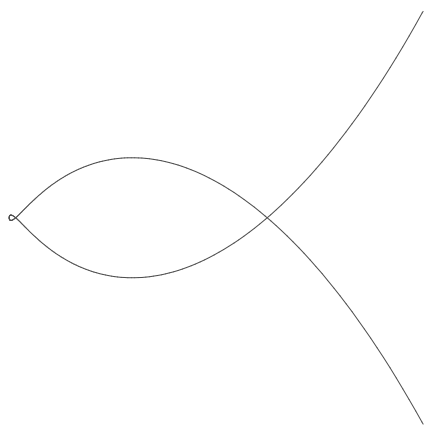

$r=1.00$
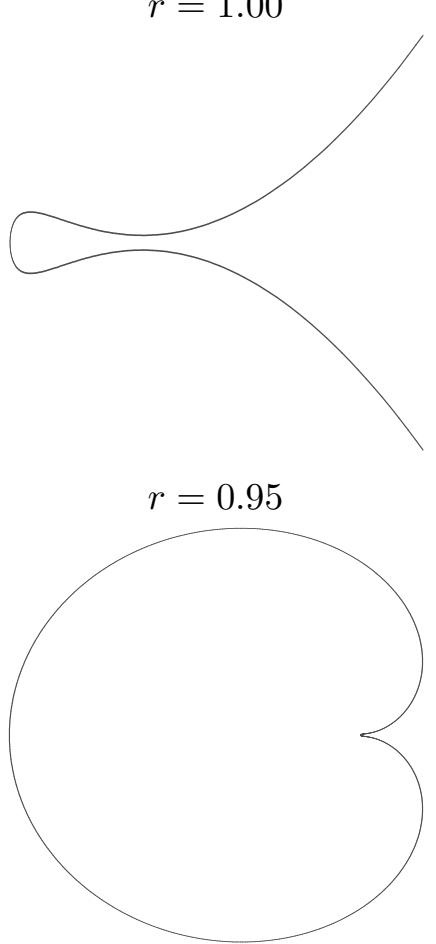

Full map $r=0.90$

Figure 2 
loop "winding in the negative sense around one or more of the branch points of the Riemann surface $W$." How can we prevent this from occurring? If $w=f(z), z=e^{i \theta}$, and $T\left(e^{i \theta}\right)$ is the tangent vector to the image curve of $C$ at $f\left(e^{i \theta}\right)$ we have the identities:

$$
T\left(e^{i \theta}\right)=(d / d \theta)\left(f\left(e^{i \theta}\right)\right)=f^{\prime}\left(e^{i \theta}\right) i e^{i \theta},
$$

hence

$$
\left.\arg (d w / d z)\right|_{\theta=\theta_{1}}-\left.\arg (d w / d z)\right|_{\theta=\theta_{2}}=\arg \left(T\left(e^{i \theta_{1}}\right)\right)-\arg \left(T\left(e^{i \theta_{2}}\right)\right)+\left(\theta_{2}-\theta_{1}\right)
$$

for $\theta_{2}>\theta_{1}$. Thus if $\left.\arg (d w / d z)\right|_{\theta=\theta_{1}}-\left.\arg (d w / d z)\right|_{\theta=\theta_{2}}<\left(\theta_{2}-\theta_{1}\right)+\pi$ for all $\theta_{2}>\theta_{1}$, then there will be no loops in the negative direction. This is the condition to be examined.

It needs to be said before doing so that Alexander made some minor misstatements prior to arriving at the condition. He considered concentric circles $\mathrm{K}$ centered at the origin having increasing radii, and said that, first, if a point of overlap occurs, either "the curve passes through a position in which it has a cusp or ... it makes one of the negative loops in question". But if in the series shown in Figure 2 the 1.1 were replaced by, say, .5 then the image of $|z|=.5$ would contain a single positive loop without having passed through a cusp on the way. Here we would have to replace "cusp" by "critical point". Secondly, he said that if a negative loop appears at any stage, it cannot disappear as the radii of the circles increase "unless the curve goes through a position in which it has a cusp". This statement is clearly wrong as the images in Figure 2 show. Again, replacing "cusp" by "critical point" would be better. In any event, his argument is not affected by either statement.

To guarantee nonnegative looping, Alexander imposed the more stringent condition $\left.\arg (d w / d z)\right|_{\theta=\theta_{1}}-\left.\arg (d w / d z)\right|_{\theta=\theta_{2}} \leq \pi$ for all $\theta_{2}>\theta_{1}$ on $C$. Writing $d w / d z=c\left(z-w_{1}\right) \ldots\left(z-w_{n-1}\right)$ it is clear that this new condition will be met if "the sum of the angles subtended at the roots of $d w / d z$ by the tangents from these points to the unit circle is less than or equal to $\pi . "$, the tangent lines giving the maximal argument change. Thus by driving the critical points outside $|z|=\csc (\pi / 2(n-1))$ his condition is fulfilled. This radius is of course greater than $\csc (\pi / n)$, which would be the optimal inner boundary of the region suggested by $w=(z-\csc (\pi / n))^{n}$, and Alexander noted this deficiency: "... the twist at the point of maximum twist on the unit circle tends to increase as the roots of $d w / d z$ approach the unit circle and ... it tends to be greater when the roots are grouped together ... It is therefore highly probable that the region $\ldots$ is determined by the function $\left[w=(z-\csc (\pi / n))^{n}\right]$ 
and consists of the points outside the circle of radius $1 / \sin (\pi / n)$ about the origin as center."

We will return to the question of the optimal region for critical points, but let us first point out that what Alexander had really done here was to define a new class of univalent functions $w=f(z)$ by the condition that $d w / d z$ has boundary values in a half plane with boundary through the origin, and he had described a region for the critical points of a polynomial of degree $n$ which ensures that the polynomial is in this class. In addition, his region for this class is maximal, as the function $w=(z-\csc (\pi / 2(n-1)))^{n}$ placed in his argument shows. If one applies the above condition to a general function, univalence is established essentially by noting that the boundary curve can't "turn back on itself" giving a "global" failure of univalence, and positive looping would not occur, since such a loop would, upon contraction of domain circles centered at the origin, pass through the image of a critical point, and by the condition there are no critical points in $|z|<1$. Such functions thus have a special geometric property: the image of any circle centered at the origin has no "reverse hairpin turns". The "standardized" version of this class, the functions $w=f(z)$ for which $\Re\left(f^{\prime}(z)\right)>0$ in $|z|<1$, is called the class of "bounded turning", since, for example, the image of a square with sides parallel to the axes is transformed into a shape which hasn't rotated from the original orientation by more than $\pm \pi / 2$ radians, as can be seen from the Cauchy-Riemann equations. He considered this class in general in $\S 6$.

As for the question of the maximal region conjectured by Alexander, it was first noticed by Kakeya [68] in 1917 that this problem is related to the Grace-Heawood theorem: If $f(z)$ is a polynomial with $f(a)=f(b)=0$, then inside the circle centered at the midpoint of the segment $a b$ and having radius $(1 / 2)|a-b| \cot (\pi / n)$ there is at least one critical point of $f$. This theorem was proved by J. H. Grace [51] in 1902, then again independently by P. J. Heawood in 1907 [58]. Van Vleck's survey amusingly calls it an "important and strange theorem" [151, p. 649]. Kakeya was attempting a generalization of this result, and relied on Heawood's method, which unfortunately was flawed ${ }^{2}$. Kakeya employed this method in his generalization, and applied "this result to the problem left unsolved by Mr. J. W. Alexander, II" [68,

\footnotetext{
${ }^{2}$ Heawood had a technique involving moving the critical points of a polynomial which vanished at \pm 1 further away from the origin and also closer together until they coincided in a single point, while maintaining the property that the polynomial thus formed still vanished at \pm 1 . Based on this procedure Heawood concluded that such a polynomial of degree $n$ must have at least one critical
} 
p. 13] as a special case to obtain the region $|z|>\csc (\pi / n)$ in which critical points may lie to assure the univalence of the polynomial of degree $n$. The result is now known as Kakeya's Theorem.

The problem was resolved in an important paper of Szegö in 1922 [143]. Gabor Szegö (1895-1985) was a prolific Hungarian analyst whose collected works include over one hundred and thirty articles and several influential books. When only 31 he was appointed professor in Königsberg, and much later he moved to the United States to head the Mathematics Department at Stanford University. His main research interests were in Toeplitz matrices, orthogonal polynomials (he produced a classic book on the subject in 1939), and special functions. The two-volume problem book [110], co-authored with G. Pólya and appearing in 1925, had a large impact on the mathematical community. Again it is interesting to find some work of such a powerful mathematician devoted to questions of polynomial theory.

In his 1922 paper Szegö noted that, with the Grace-Heawood theorem in hand, we can allow $a$ and $b$ to be arbitrary points in $|z| \leq 1$ and construct the envelope of points $P$ of the form $P=(a+b) / 2+e^{i \theta}(|a-b| / 2) \cot (\pi / n)$ as 0 varies from 0 to $2 \pi$, and be guaranteed that as long as all critical points of $f$ lie outside this envelope, we cannot have $f(a)=f(b)$ in the unit circle. The envelope is easily computed to be $|z| \leq \csc (\pi / n)$, "allowing us without great difficulty to derive a theorem of Alexander and Kakeya" [p. 53]. (A similar approach was taken in Robertson's first publication [119] in 1932.) Szegö's paper was important for several reasons, partly because of the introduction of convolution ideas into univalent function theory. We will mention many of his results in the next section, but note here that he proved, for example, that if $f(z)=\sum_{n=1}^{N} a_{n} z^{n}$ and $g(z)=\sum_{n=1}^{N} b_{n} z^{n}$ are univalent polynomials on $|z| \leq 1$, then $h(z)=\sum_{n=1}^{N}\left(\begin{array}{c}N-1 \\ n-1\end{array}\right)^{-1} a_{n} b_{n} z^{n}$ is also univalent in $|z| \leq 1$. This is perhaps the first instance of a theorem involving the univalence of the convolution of two univalent polynomials, a line of thought which was revived in the 1950's.

Let us conclude this section by returning to Biernacki's thesis. Biernacki $[15, \mathrm{p}$. 660] considered Alexander's conjecture as the beginning of a chain of results which consisted of successive generalizations of Lucas's theorem: if a circle contains all $n$ zeroes of a polynomial, then it contains also the $n-1$ zeroes of the derivative. The first generalization, he claimed, was obtained by Kakeya in the paper mentioned

point inside the circle $|z|=\csc (\pi / n)$. But an examination of his method shows that the argument is invalid, as was pointed out by Başgöze in a 1968 paper [6]. 
above: if a circle of radius $R$ contains $p$ zeroes of a polynomial of degree $n$, then a concentric circle of radius $\Psi(n, p) \cdot R$ contains at least $p-1$ zeroes of the dervative, for some function $\Psi$. He pointed out that Kakeya calculated $\Psi(n, 2)=\csc (\pi / n)$, stating "This result had previously been cited as a probable by M. Alexander..." [p. 660], and quoted Szegö's work as well as that of some others. Biernacki himself considered the case $p=n-1$. In this instance Alexander's interest in univalent polynomials influenced some work in general polynomial theory.

\section{Convolutions}

The two problems considered by Alexander in $\S 4$ and $\S 5$ were among the first in univalent function theory to be treated by the method of convolutions, a method which has since become important in the theory. Let us depart from the contents of Alexander's paper and detail how this treatment came about. In the paper by Szegö mentioned above, the author provided a new proof of a theorem of Grace regarding the apolarity of polynomials. Two polynomials $f(z)=\sum_{k=0}^{n} a_{k} z^{k}$ and $g(z)=\sum_{k=0}^{n} b_{k} z^{k}$ are said to be "apolar" if $a_{0} b_{n}-C(n, 1) a_{1} b_{n-1}+C(n, 2) a_{2} b_{n-2}-$ $\cdots+(-1)^{n-1} C(n, n-1) a_{n-1} b_{1}+(-1)^{n} a_{n} b_{0}=0$, where $C(n, k)$ is the $k$ th binomial coefficient. This rather odd-looking condition comes about by considering the repeated derivatives of these polynomials with respect to the zeroes of the other; this classical turn-of-the-century material was laid out in the Pólya-Szegö problem book [110, Vol 2, p. 59]. Grace's theorem, from which his Rolle-like result mentioned in the previous section follows, is that if two polynomials are apolar, then any circle containing all the zeroes of one polynomial contains at least one zero of the other. This result appeared in [51]. From this theorem Szegö obtained a convolution result: let the zeroes of the polynomials $f(z)=\sum_{k=0}^{n} C(n, k) a_{k} z^{k}$ lie in a circular domain $C$, and $g(z)=\sum_{k=0}^{n} C(n, k) b_{k} z^{k}$ of degree $n$ have zeroes $\left\{B_{k}\right\}$, then any zero $q$ of $h(z)=(f * g)(z)=\sum_{k=0}^{n} C(n, k) a_{k} b_{k} z^{k}$ can be written as $q=-\left(B_{k}\right) P$ for some $k$, where $P$ is a point in $C$. In addition to this convolution result he derived the Grace-Heawood Theorem by noting that the condition $f(1)=f(-1)$ imposes a condition on the coefficients of the polynomial $f$ which implies that it is apolar to $g(z)=(z-1)^{n}-(z+1)^{n}$, where $n$ is the degree of the polynomial $f$. But the zeroes of $g$ are at $-i \cot (k \pi / n), k=1 \ldots n$. Thus by Grace's apolarity theorem the circle 
centered at zero having radius $\cot (\pi / n)$ contains a zero of $f^{\prime}$. Grace had also made this observation in $[51, \text { p. } 356]^{3}$.

Dieudonné in his thesis (pp. 313-315) used Szegö's convolution theorem to deduce the two results of Alexander and Kakeya in $\S 4$ and $\S 5$ of [2]. He first introduced what has become known as the "Dieudonné criterion" for the univalence of polynomials: if $f(z)=z+a_{2} z^{2}+\cdots+a_{n} z^{n}$, then the radius of univalence of $f$ is the radius of the largest circle centered at the origin containing no root of $F(x, t)=1+$ $a_{2} x \sin (2 t) / \sin (t)+a_{3} x^{2} \sin (3 t) / \sin (t)+\cdots+a_{n} x^{n-1} \sin (n t) / \sin (t)$, for any $t, 0<$ $t<\pi$; this criterion follows by considering the condition $\left(f\left(x e^{i t}\right)-f\left(x e^{-i t}\right)\right) /\left(x e^{i t}-\right.$ $\left.x e^{-i t}\right) \neq 0$ for all $|x|<1$ and $0<t<\pi$. From this criterion and Szegö's convolution result he deduced "immediately" the following theorem: let $f(z)=z+\sum_{k=0}^{n} a_{k} z^{k}$ and $g(z)=z+\sum_{k=0}^{n} b_{k} z^{k}$ have $R$ and $R^{\prime}$ respectively as radii of univalence. Then we have $L R^{\prime} \leq R$, where $L$ is the magnitude of the zero of smallest modulus of $H(z)=1+C(n-1,1)\left(a_{2} / b_{2}\right) z+\cdots+\left(a_{n} / b_{n}\right) z^{n-1}$. This is a version of Szegö's convolution theorem for univalent polynomials, and Dieudonné noted the similarity.

Dieudonné used this theorem to deduce both of Alexander's results as follows. He first set $b_{2}=C(n-1,1), \ldots, b_{n-1}=C(n-1, n-2), b_{n}=1$ to get $g(z)=z(1+z)^{n-1}$ and $H(z)=f(z) / z$. We have seen before that $R^{\prime}=1 / n$, and consequently if $z_{0}$ is the zero of smallest modulus of $f(z) / z$, then $f$ is univalent in the circle $|z| \leq\left|z_{0}\right| / n$. This is Alexander's result of $\S 4$, although lacking the proof of the starlikeness of $f$. As for the conjecture in $\S 5$, if we take $b_{2}=C(n-1,2) / 2, b_{3}=C(n-1,3) / 3, \ldots, b_{n}=1 / n$, then we get $g(z)=\left((1+z)^{n}-1\right) / n$, and $H(z)=f^{\prime}(z)$. Thus $R^{\prime}=\sin (\pi / n)$, and if $z_{1}$ is the critical point of $f$ closest to the origin, then $f$ is univalent in $|z| \leq\left|z_{1}\right| \sin (\pi / n)$, giving Kakeya's theorem. These results were reproduced in Montel's volume [95].

This use of a technique of convolution is a good example of the material in "the overlapping region belonging alike to the theory of equations and that of analytic functions" mentioned by Van Vleck in his 1929 survey [151, p. 643] of these results of Grace and Szegö from the first quarter of the $20^{\text {th }}$ century. A 1931 paper of L. Teodoriu [147] moved further into the domain of function theory by considering

\footnotetext{
${ }^{3}$ In the same year as Szegö's paper E. Egerváry published work [33] related to the same set of problems. He considered a general maximum-minimum problem for a multilinear relation of complex constants whose solution contained the Grace-Heawood result as a special case, and also dealt with apolarity of polynomials in this context. Szegö and Egerváry refer to each other's work in these papers. Egerváry was a mathematician of the Hungarian school whose work included complex function theory and combinatorics.
} 
the entire function $f(z)=z\left(z-z_{1}\right)^{n_{1}} \ldots\left(z-z_{k}\right)^{n_{k}} e^{g(z)}$ for $g$ analytic in the plane. He produced a real $\phi(k, M)<1 / k$ such that there are no critical points for $f$ in $|z|<\phi(k, M)$, where $M=\max _{z \in C}\left|g^{\prime}(z)\right|$ for a contour $C$ containing $\left\{z_{k}\right\}$. If $g(z)=0$ he pointed out that his result gives the same region as Alexander for univalence of $f$; he, however, did not prove univalence. That Grace's and Szegö's theorems were considered topics in algebra at this time can be seen by their inclusion in Bieberbach's 1928 algebra text [13].

As mentioned earlier, Dieudonné and Szegö each devoted more extended work to these polynomial problems. In 1932 Szegö reported that, in a conversation with Leopold Fejér [145, p. 237], the question of radii of (1) univalence, (2) starlikeness, and (3) convexity of polynomials with known zeroes and critical points arose. If $P(z)=z+a_{2} z^{2}+\cdots+a_{n} z^{n}$ and $d$ is the distance to the nearest zero, $D$ the distance to the nearest critical point, then of the radii $r_{1}, r_{2}, r_{3}$ corresponding to $d$ and $R_{1}, R_{2}$, and $R_{3}$ corresponding to $D$ he noted that only $R_{2}$ and $r_{3}$ were unknown, the others having been determined by Alexander and Kakeya. He found the values of $R_{2}$ and $r_{3}$ by apolarity methods. In 1934 [30] Dieudonné generalized some of these results by finding the radius of convexity and starlikeness for $z[P(z) / z]^{(a / n)}$, for $a$ any real number and considered related problems. His methods were distinguished by the use of recently developed facts in function theory related to growth estimates for functions of bound one in the unit disc (See appendix $[\mathbf{A 1}]$.)

\section{More Function Classes}

Let us return to Alexander's paper. In $\S 6$ he began by pointing out that the condition in $\S 5$ which assured that his polynomials are univalent, namely that "the range of variation of $d w / d z$ on the unit circle is less than $\pi$, or in other words that the function $w^{\prime}=d w / d z$ maps the interior of the unit circle upon a region contained within a half plane bounded by a straight line through the origin" [p. 18], is a condition which can be used to define a class of univalent mappings. He noted that coefficient conditions on a function mapping the unit disc into such a half plane had already been studied by Carathéodory in [24], in 1907. In this paper Carathéodory described the "sections" of functions $w=1+A_{1} z+A_{2} z^{2}+\cdots$ of positive real part, showing that the coefficient body $\left\{A_{1}, A_{2}, \cdots, A_{n}\right\}$ in $2 n$ dimensional space consists of the smallest convex $2 n$-dimensional body which contains the curve $(2 \cos (t),-2 \sin (t), 2 \cos (2 t),-2 \sin (2 t), \ldots, 2 \cos (n t),-2 \sin (n t))$, as $t$ ranges from 0 to $\pi$. Other contemporary studies of this problem included 
a 1911 paper in which Carathéodory characterized the same class of mappings in terms of the Carathéodory-Toeplitz determinant condition, and the 1911 Herglotz result which gives the class as the well-known integral of $\left(e^{i t}+z\right) /\left(e^{i t}-z\right)$ against a probability measure (see appendix $[\mathbf{A 2}]$ ).

In the remainder of $\S 6$ Alexander provided a simple sufficient coefficient condition for membership in the bounded turning class, which we will rewrite slightly: if $w=a+a_{1} z+a_{2} z^{2}+\cdots$ is analytic in the unit disc, we impose the condition $\left|a_{1}\right| \geq\left|2 a_{2}\right|+\left|3 a_{3}\right|+\cdots+\left|n a_{n}\right|+\cdots$. From this condition bounded turning will follow, since $\left|a_{1}\right| \geq \sum_{k=2}^{\infty}\left|k a_{k}\right| \geq\left|\sum_{k=2}^{\infty} k a_{k} z^{k}\right|=\left|d w / d z-a_{1}\right|$ for $z$ in the disc. Thus " $d w / d z$ always lies in the half-plane bounded by the normal to the vector $a_{1}$ through the origin and containing the point $a_{1}$ ". He achieved the goal of providing a useful criterion in terms of the function's coefficients that its mapping be one to one.

Looking ahead into the last section of his paper, $\S 9$, he returned to the condition $\sum_{k=2}^{\infty}\left|k a_{k}\right| \leq\left|a_{1}\right|$ and attempted to show that it also assures that the mapping is starlike. This he did by writing $(d / d \theta) \arg w \geq\left|a_{1}\right||(d / d \theta) \arg z|-$ $\sum_{k=2}^{\infty}\left|a_{k}\right|\left|(d / d \theta) \arg \left(z^{k}\right)\right| \geq\left|a_{1}\right|-\sum_{k=2}^{\infty}\left|k a_{k}\right| \geq 0$. The fallacy in this proof is clear, since $\arg (z)$ is not an additive function, but it is a remarkable fact that the theorem as stated is true. The first proofs were offered independently in 1932 by Noshiro [99], who dealt with the starlike class, and Kobori [76], who dealt with the corresponding result for the convex class. (Noshiro's work on the problem appears not to have been noted in the literature.) In the first extensive analysis of the class of functions $f(z)=\sum_{n=1}^{\infty} a_{n} z^{n}$ for which $\sum_{k=1}^{\infty} k\left|a_{k}\right| \leq\left|a_{1}\right|$, given by Remak [118] in 1943, the author proved the starlikeness of the mappings, and stated that "Herr Bieberbach pointed out in his day the inadequacy of this [Alexander's] proof" [p. 176]. Remak himself had published in Bieberbach's time; [117] is an example of his work in analysis in this era.

Alexander concluded $\S 9$ with the corollary that a mapping satisfying $\sum_{k=2}^{\infty} k^{2}\left|a_{k}\right|<$ $\left|a_{1}\right|$ maps the unit disc univalently onto a convex domain. This last result was quoted in the review [64], and perhaps this accounts for the attribution by later writers of these two theorems to Alexander. It is possible that Alexander was led to the conjecture by noting that the polynomials $z-z^{n} / n$, which are univalent by his criteria, map the unit circle onto epicycloids with cusps, which by construction can be seen 
to be starlike. He considered these polynomials in the $\S 7$ in an analysis of their critical points.

It is fruitful to consider these sections as the beginning of the exploration of several new classes of normalized univalent functions. For $f(z)=z+a_{2} z^{2}+\cdots$ analytic in the unit disc we have $S^{*}$, the starlike functions; $B$, the class of bounded turning; $R$, the class for which $\sum_{k=2}^{\infty}\left|k a_{k}\right|<1$, and the class $\mathrm{M}$ (introduced and studied by MacGregor in his thesis (1961) and [86]) of functions for which $\left|f^{\prime}(z)-1\right| \leq 1$. Alexander proved the class inclusions $R \subset M \subset B$, and argued for $R \subset S^{*}$. Each of these classes and class inclusion questions was studied in detail by later authors, in such papers as [118], [97] and [86]. A fifth class, $N$, of functions with $\mid z f^{\prime}(z) / f(z)-$ $1 \mid<1$ needs to be mentioned, since Noshiro's 1932 result is the inclusion $R \subset N$ (note the similarity to Alexander's proof that $R \subset B$ ) and clearly $N \subset S^{*}$. Noshiro's proof was rediscovered by Lewandowski [81] in 1955, and Clunie and Koegh [26] in 1960: $\left|z f^{\prime}(z)-f(z)\right|-|f(z)|=\left|\sum_{k=2}^{\infty}(k-1) a_{k} z^{k}\right|-\left|z+\sum_{k=2}^{\infty} a_{k} z^{k}\right|<\sum_{k=2}^{\infty}(k-$ 1) $\left|a_{k}\right|-\left(1-\sum_{k=2}^{\infty}\left|a_{k}\right|\right)=\sum_{k=2}^{\infty}\left|k a_{k}\right|-1 \leq 0$, thus dividing through by $|f(z)|$ gives the result. Goodman [47] provided an alternative argument in 1957.

Let us finish by returning to Dieudonné's query at the end of our section on starlike and convex polynomials. Dieudonné mentioned that the class $R$ introduced by Alexander is a class for which the radius of univalence, radius of starlikeness, and minimum magnitude of critical points are all equal to one. In answer to his question of whether these three numbers always coincide for a given class of functions, we may point out that the bounded turning class in general does not exhibit this coincidence. Although the radius of univalence for the class is clearly 1 , and the examples $f(z)=$ $z+z^{n} / n$ show that the class can have members with critical points on the boundary, there are examples of bounded turning functions which are not starlike on the unit disc, showing that the radius of starlikeness need not be equal to 1 . In [112], for instance, it is noted that $f_{a}^{\prime}(z)=1+\frac{z(a+z)}{1+a \bar{z}}$ provides such an example.

\section{History Surrounding the Alexander-Noshiro-Kobori Theorem}

The Alexander-Noshiro-Kobori result has a history which predates Alexander's work. At the International Mathematical Congress in Zurich in 1897 Hurwitz presented a paper "On the Development of the General Theory of Analytic Functions in Modern Times" in which he surveyed various aspects of analytic function theory. At one point, while discussing the theory of singularities, he noted that there was not a developed theory for non-isolated singularities: "Generally, to my knowledge, 
a thorough study of the behavior of an analytic function in the neighborhood of non-isolated singularities is not at hand. However, there is a relevant fact that one can mention ..." [152, p. 99] He then went on to discuss the work of several mathematicians in this area, among whom was I. Fredholm, and remarked on the existence of power series which are not analytically continuable across the boundary of the circle of convergence. Furthermore: "One can now form an example of [such a] power series, for which not only the analytic function, but also all its derivatives are continuous in the convergence circle and on the boundary" [p. 99].

In a footnote of the conference proceedings [152] he noted that Fredholm gave an example of a power series, namely $f(z)=1+a z+a^{2} z^{4}+\cdots+a^{n} z^{n^{2}}+\cdots,|a|<1$ which is not analytically continuable across the boundary of the unit circle by the recently proved Fabry Gap Theorem. (The series is clearly $C^{\infty}$ on the unit circle.) Hurwitz then reported: "In a conversation during the Congress Herr Fredholm called attention to the fact that the inverse of the function $f(z)$ is, for a suitable choice of the constant $a$, a single-valued function. In that case, if $|x|<1,|y|<1$, one finds easily that $|(f(x)-f(y)) /(x-y)|=\left|\sum a^{n}\left[x^{n^{2}-1}+x^{n^{2}-2} y+\cdots+x y^{n^{2}-2}+y^{n^{2}-1}\right]\right|>$ $|a|-4\left|a^{2}\right|-9|a|^{3}-\cdots-n^{2}|a|^{n}-\cdots$ and from here it follows that $f(x)=f(y)$ can only occur when $x=y$, if only $|a|$ is sufficiently small" [p. 109]. (A value of $a \approx 0.16$ will do.) The proof of univalence is clearly similar to Alexander's and the condition which assures a simple mapping is the same, but it is worth noting that only univalence is proved; no geometric mapping properties are inferred from the condition. Also, Alexander stated the theorem in general, that is, not as a method applied to a particular example.

In 1898 W. F. Osgood published an example [103] of a similar univalent noncontinuable series, but the proof of univalence was quite complicated. However, the method Fredholm used on his series was applicable to Osgood's: in a note several months later Osgood [104] reported "I am indebted to Professor Hurwitz for an exceedingly simple proof of the principal theorem of my note, namely, that the inverse function is single valued ... This follows at once by the application of a method employed by Professor Fredholm ..." [p. 17]. Osgood's example was $f(z)=z+\sum z^{a^{k}+2} /\left(a^{k}+1\right)\left(a^{k}+2\right)$; he mentioned that $f$ and its first derivative are continuous in the unit disc and on the boundary. 


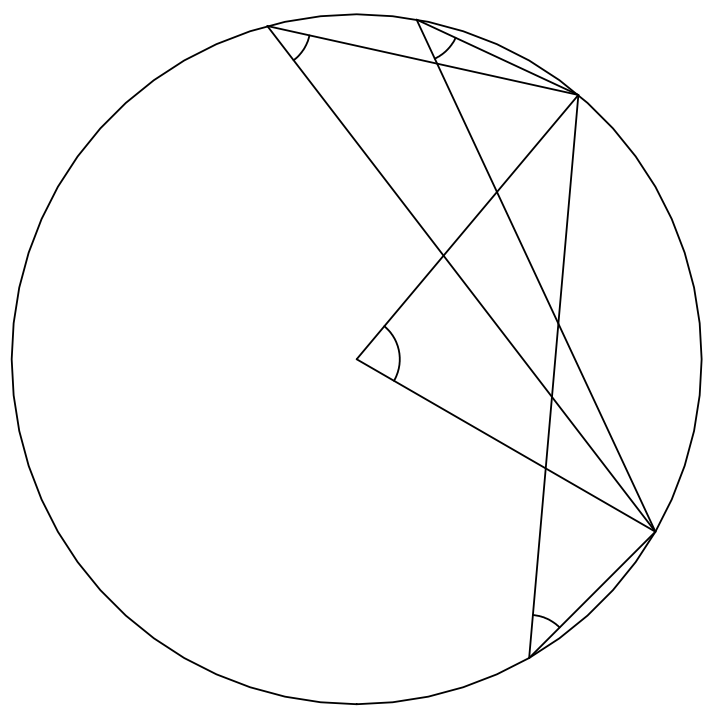

Figure 3

Osgood pointed out in his first paper that univalent mappings of the unit disc which have the unit circle as natural boundary can easily be shown to exist on theoretical grounds, but that specific examples are always of interest. "I have thought it worthwhile to call attention to the existence of functions of the kind I have treated, because they are useful in theoretical investigations. To cite a single instance: the theorem that an analytic function of an analytic function is an analytic function is true im Kleinen [locally], but not necessarily im Grossen [globally]" [p. 422]. Im Kleinen analyticity of a function is equivalent to analyticity in a neighborhood of a point. Im Grossen analyticity involves the analytic continuation of a function defined on a given open set to another analytic function defined on an open set disjoint from the first. Osgood's example of the failure of im Grossen analyticity of a composition uses his noncontinuable series to construct two locally analytic functions whose composition is not analytically continuable from one component of its domain to the other. (See appendix $[\mathbf{A 3}]$.)

\section{Critical Points On The Boundary}

Returning now to $\S 7$, Alexander considered the possibility of univalent polynomials with critical points on the boundary of the unit circle, which he showed can occur provided that "they [the critical points] are not too close to one another". 


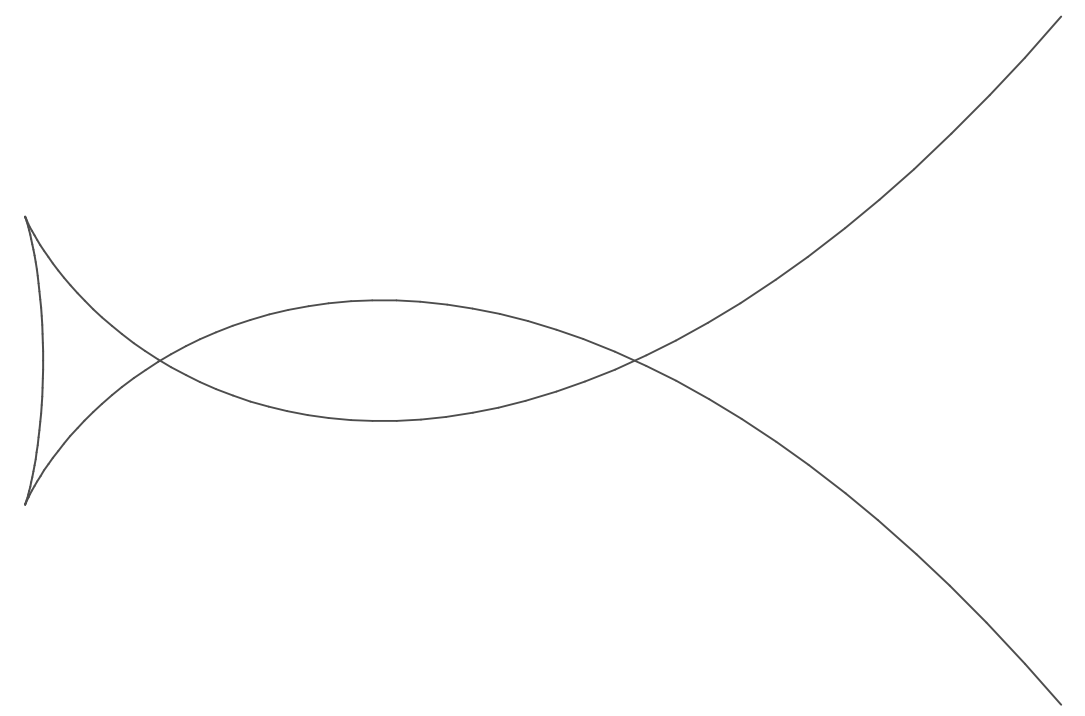

Figure 4: Cusp

$$
f(z)=z-\left(\cos (\pi / 10) z^{2}+(1 / 3) z^{3}\right.
$$

He first noted that if the critical points $w_{1}, w_{2}, \ldots, w_{n-1}$ of a polynomial $P_{n}(z)$ of degree $n$ lie in order on the unit circle, then each arc $w_{k} w_{k+1}$ is mapped on to a curve of positive curvature. This can be seen by referring to Figure 3, for example, and writing $P_{n}^{\prime}\left(e^{i t}\right)=c_{n} \prod_{k=1}^{n-1}\left(e^{i t}-w_{k}\right)$, then the argument of the tangent vector to $P_{n}\left(e^{i t}\right)$ equals $\arg \left(i e^{i t} P_{n}^{\prime}\left(e^{i t}\right)\right)=\pi / 2+t+\arg c_{n}+\sum_{k=1}^{n-1} \arg \left(e^{i t}-w_{k}\right)$; this is clearly an increasing function of $t$ as $e^{i t}$ moves counterclockwise between critical points. (Alexander didn't give this much detail, he merely said that “... a point in describing the arc turns in a positive sense about all the points $w_{1}, w_{2}, \ldots, w_{n-1}$ ".) "[The image of the unit circle] therefore consists of $n-1$ arcs all concave inward and abutting in pairs at the branch points of the Riemann surface ... at each of which they form a cusp."

The next part of the argument is to show that if the tangent vector increases by at least $\pi$ between cusps, then the image of the unit circle cannot intersect itself. By insisting that the tangent vector turn be greater than $\pi$, we avoid the possibility of "global" self intersection as shown for example in Figure 4. Then the only remaining possibility is the existence of a "... simple loop winding in the positive sense about the region which it enclosed." But this can be ruled out because, by considering the images of concentric circles centered at the origin of radii smaller than 1 and decreasing to zero, we find that they too must contain such a loop, which can only 


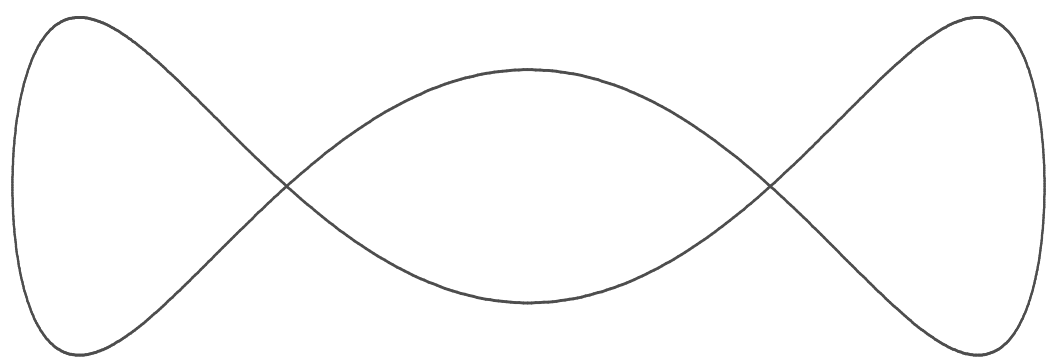

Figure 5

disappear by "shrinking to a cusp", an impossibility, since all the critical points are on the boundary.

Before continuing to consider the amount by which the critical points must be separated to ensure the required tangent vector turn, we should consider some difficulties. As we noted before, a curve containing a simple loop in the positive sense can shrink down and have the loop disappear without passing through a critical point, recall Figure 2. In fact this type of resolution of a loop can occur if the function has no critical points at all, as can be seen by examining the function $e^{z}$ on circles centered at the origin and having decreasing radii. Furthermore, Alexander had not considered the kind of global self intersection shown in Figure 5, which, however, could not occur due to negative winding numbers in some of the components of the complement of the curve. It is also possible for a curve to contain subarcs of argument turn greater than $\pi$ and still contain a simple positive loop. Suffice it to say that the possibilities for self intersection need to be more comprehensively analyzed.

Referring to Figure 3 and the expression above for the argument of the tangent vector, we see that if the angle between two critical points on the circle is $\theta$, then the change in the argument of the tangent vector as we go from one critical point to the next is $\theta+\left[\arg \left(w_{2}-w_{1}\right)-\arg \left(\right.\right.$ tangent at $\left.\left.w_{1}\right)\right]+\left[\arg \left(-\right.\right.$ tangent at $\left.w_{2}\right)-\arg \left(w_{1}-\right.$ $\left.\left.w_{2}\right)\right]+\sum_{k=3}^{n-1}\left[\arg \left(w_{2}-w_{k}\right)-\arg \left(w_{1}-w_{k}\right)\right]$. Each of the argument changes in brackets equals $\theta / 2$, making a total of $(n+1) \theta / 2$ for the tangent vector change. Thus, according to Alexander's reasoning, the curve cannot cross itself if $\theta>2 \pi /(n+1)$. This is the desired minimal separation value for critical points lying on the unit circle which ensures univalence of the mapping polynomial. Again, it is assumed that univalence on the boundary implies univalence in the interior. He closed the section by giving three examples of polynomials whose critical points satisfy the 
separation criteria with the following minimal separation values: $f_{1}(z)=z-z^{n} / n$ with $2 \pi /(n-1), f_{2}(z)=z+z^{2} / 2+z^{3} / 3+\cdots+z^{n} / n$ with $2 \pi / n$, and $f_{3}(z)=$ $z+z^{3} / 3+z^{5} / 5+\cdots+z^{2 k+1} /(2 k+1)$ with $2 \pi /(n+1), n=2 k+1$. See [46] for a detailed treatment of $f_{3}$; Alexander made a slight error in accounting for the location of the critical points.

The three polynomials mentioned by Alexander have interesting phenomena associated with them. The first is the epicycloid which in the previous section was seen to be both starlike and of bounded turning. W. F. Osgood in [105] detailed the construction of Riemann surfaces for $f_{1}$ in the cases $n=3$ and $n=4$, noting that the case $n=3$ had been dealt with by Felix Klein in his Leipziger Vorlesung, 1881-1882. (Klein's careful treatment of this example can be found in [75, pp. 7077].) Perhaps these constructions came to Alexander's attention. Remak in 1943 [118] proved that for functions $f$ in the class $R$ for which $a_{p}$ is the first non-zero coefficient, we have $1-1 / p \leq\left|f\left(e^{i \theta}\right)\right| \leq 1+1 / p, 0 \leq \theta \leq 2 \pi$, with the extremal functions being $f(z)=z \pm z^{p} / p$. In 1970 [19] it was proved that these polynomials are in fact the only starlike polynomials of their degree with all critical points on the unit circle; the starlike requirement forces the extremal degree of separation.

The second and third mappings are closely related to topics in Fourier series, one of which may have been brought to Alexander's attention by T. H. Gronwall. These topics were the province of Leopold Fejér (1880-1959), leader of the Hungarian school of analysis from his appointment to the chair of mathematics at the University of Budapest in 1911 until his death in 1959. His main work was in harmonic analysis, power series and Fourier series, and his name is most closely associated with a classical summation theorem for the Fourier series of a continuous function; this topic formed the basis for his doctoral thesis in 1902.

At the turn of the twentieth century and in its first decade much study was made of the convergence properties of Fourier series, the Gibbs phenomenon, and continuous functions with divergent Fourier series. In these studies an important role was played by the series $\sum_{n=1}^{\infty} \sin (n t) / n$ and $\sum_{n=1}^{\infty} \sin ((2 n-1) t) /(2 n-1)$, which are the infinite series corresponding to the imaginary parts of $f_{2}$ and $f_{3}$ on the unit circle. The first of these series is the Fourier series for $f(x)=(1 / 2)(\pi-x)$ on $0<x<\pi$. In 1911 Fejér [36] proved that the partial sums of the first series are uniformly bounded by the constant $(\pi / 2)+1$, a special case of the theorem that 
any series $\sum_{n=1}^{\infty} a_{n} \sin (n t)$ with $a_{n}$ positive, decreasing, and $n a_{n}$ bounded is itself boundedly convergent; see ([57, p. 29]).

In 1899 Gibbs pointed out the "overshoot" effect of the partial sums of the similar series $\sum_{n=1}^{\infty}(-1)^{n+1} \sin (n t) / n$, without supplying any proof; it remained until 1906 for Bôcher to prove a generalization of this observation, namely that under mild restrictions on a real valued function, the partial sums of its Fourier series exhibit the "Gibbs' overshoot" at any finite jump. (See [25] for other historical precedents, exact statements, and references). Bôcher's general proof relied on the fact that the phenomenon could be demonstrated for $\sum_{n=1}^{\infty} \sin (n t) / n$ first.

In 1910 Fejér conjectured that the partial sums of $\sum_{n=1}^{\infty} \sin (n t) / n$ are all positive in the interval $0<t<\pi$, perhaps motivated by seeing graphs of the Gibbs phenomenon for this function. The first proof of this fact was given by D. Jackson in a paper [62] dated February 1911. Dunham Jackson (1888-1946) was an American mathematician chiefly known for his outstanding contributions to approximation theory; his theorems relate to the approximation of continuous real functions by polynomials and trigonometric sums. As in the case of Nevanlinna, Robertson, and Dieudonné, Jackson's relevant publication appeared nearly simultaneously with his doctoral dissertation. Jackson's paper was closely followed by a proof given by Gronwall in a paper dated April 1911 [52]. The relation between this result and Alexander's is clear: Alexander's univalence result implies a strengthening of the nonnegativity of the partial sums. This is intuitively clear but can also be made precise by invoking the Dieudonné univalence criterion: from Alexander's result it follows that $\sum_{k=1}^{n} z^{k-1} \sin (k t) / k \sin (t) \neq 0,|z|<1,0<t<\pi$, and all $n$; now letting $z$ real approach 1 gives the required non-negativity. (See [3], where this approach is credited to J. Bustosz.)

Going in the other direction, Fejér used known facts about non-negative trigonometric sums to offer an alternative proof of the univalence of $f_{3}$. In a "lecture delivered in May 1933 at several universities in the eastern and Middle western States" [39] Fejér presented among other topics his result that the third arithmetic mean of a vertically convex univalent function (a three-fold averaging of the partial sums of the Taylor series for the function) is itself univalent throughout the unit disc. (See [46] for a discussion of this theorem.) In doing so he gave two examples to motivate the discussion of the univalence of partial sums of a univalent function: the function $1 /(1-z)$, each of whose partial sums fails to be univalent 
in $|z|<1$, and $(1 / 2) \log ((1+z) /(1-z))$, whose partial sums are $f_{3}$, and are all univalent throughout $|z|<1$. He credited the univalence of $f_{3}$ to Alexander, and also supplied an alternative proof: in writing $f_{3}=u(t)+i v(t)$, he noted that $v(t)=\sum_{k=1}^{n} \sin ((2 k-1) t) /(2 k-1)$ is "well-known" to have positive values on the interval $0<t<\pi$ (in fact it follows from the non-negativity of $\sum_{k=1}^{n} \sin (k t) / k$ by substituting $t+\pi$ for $t$ and combining the appropriate inequalities), and that $d u(t) / d t=-\sum_{k=1}^{n} \sin ((2 k-1) t) \leq 0$ on $0<t<\pi$. These facts prove the vertical convexity of the polynomials $f_{3}$ by demonstrating that the image of the upper half of the unit circle is a curve whose real part moves constantly to the left as the circle is traced out in the counterclockwise direction, and that the imaginary part of the image of the unit circle in the upper half plane always lies in the upper half plane. (Fejér later realized that only the requirement on the real part of a function is needed to ensure univalence). This adds another interesting geometric touch to Alexander's results. (See appendix $[\mathbf{A 4}]$.)

\section{Close-To-Convex Functions}

In $\S 8$, perhaps the richest section of the paper, Alexander introduced one final class of univalent mappings by proposing another criterion for univalence apparently motivated by the material in $\S 7$. This condition is as follows: we assume that from each point $z$ of the unit circle there stems a ray which satisfies three conditions: (a) the positive angle through which the ray must be turned in order to make it coincide with the positive tangent vector to the image curve is between 0 and $\pi$; (b) The angle $\psi$ which the ray makes with the positive real axis is a nondecreasing function of $\arg (z)$; (c) the angle $\psi$ will not increase by more than $2 \pi$ as $\arg (z)$ varies from 0 to $2 \pi$. He pointed out that (a) can be expressed as $\pi / 2 \geq \arg (z(d w / d z))-\psi \geq-\pi / 2$, i.e., the difference between the argument of the normal to the image curve and $\psi$ is between $-\pi / 2$ and $\pi / 2$, and that this implies that the ray never points to the side of the image curve corresponding to the interior of the unit circle.

That the existence of such a ray implies univalence follows as in the previous sections: Alexander argued that if the function had a critical point in the interior of the unit disc, this would cause the tangent to the image of the unit circle to rotate through an angle of $2 k \pi, k>1$, as $\arg (z)$ varied from 0 to $2 \pi$, whereupon no set of rays could satisfy (a) and (c) simultaneously. On the other hand, he pointed out that if the critical points were on or outside the unit circle, the image loop couldn't 
cross itself unless it contained a loop in the negative direction, but then (a) and (b) could not be satisfied simultaneously.

We have dealt with the difficulties of this type of argument before, and we need not repeat the objections, but let us point out that effectively what Alexander was again doing was to rule out the possibility of either positive or negative looping by his three conditions. Although he didn't mention this, he also ruled out the possibility of a decrease in the argument of the tangent vector to the image curve by more than $\pi$, as $z$ traverses the unit circle in the counter clockwise direction, even after the univalence is proved. Thus there are no "reverse hairpin turns" in the image curves. We can also note that if $\psi=\arg (z)$, then his condition reduces to the bounded turning condition considered in $\S 6$; thus we have an attempt to generalize that condition and also guarantee the distinctive characteristic of no reverse hairpin turns. In addition, if the ray can be chosen to be the normal vector to the image curve, then the mapping is convex univalent. The new class defined by Alexander was explored rigorously for the first time in 1952 by W. Kaplan [70] and called by him the "close-to-convex" class.

Alexander's purpose in introducing this condition was to provide another proof of the univalence of the functions $f_{1}, f_{2}$ and $f_{3}$ from the preceeding section by clarifying the geometric implications of the critical point placement. He proved this for $f_{2}$ by choosing the ray to be the normal vector to the convex function for which $f_{2}$ forms the partial sums, and for $f_{3}$ he chose a related function. For example, for $f_{2}$ he set $\psi=\arg (z /(1-z))$; then (b) and (c) are satisfied, and a computation shows that (a) is satisfied: $\arg (z(d w / d z))-\arg (z /(1-z))=\arg \left((1-z)\left(1+z+z^{2}+\cdots+z^{n}\right)\right)=$ $\arg \left(1-z^{n+1}\right)$. For $f_{3}$ he used $\psi=\arg ((1+z) /(1-z))$, and, though he didn't mention it, for $f_{1}$ we can use $\psi=\arg (z)$. It should be noted that for $f_{3}$ Alexander could have chosen with success the normal to the limit of the $f_{3}$ sequence, namely $\psi=\arg \left(z /\left(1-z^{2}\right)\right)$, for the calculation. Thus one way of interpreting this type of calculation is to say, in the case of $f_{2}$ and $f_{3}$, that the partial sums of $\log (1 /(1-z))$ and $\log ((1+z) /(1-z))$ are "close" to these functions in the sense that the normal vectors of the partial sums at any point don't differ by more than $\pi / 2$ from the normals to the limit functions.

Alexander next made an observation that allowed him to generalize the above results: if two mappings $w_{1}$ and $w_{2}$ both satisfy the above three conditions for the same choice of $\psi$, then so does $w_{1}+w_{2}$. This follows from the parallelogram 
law and the inequalities $\arg (z)+\arg \left(d w_{1} / d z\right)-\psi \leq \arg (z)+\arg \left(\frac{d}{d z}\left(w_{1}+w_{2}\right)\right)-$ $\psi \leq \arg (z)+\arg \left(d w_{2} / d z\right)-\psi$ where we assume without loss of generality that $\arg \left(d w_{1} / d z\right) \leq \arg \left(d w_{2} / d z\right)$. Clearly this generalizes to convex combinations of $\left\{w_{1} \ldots w_{n}\right\}$ all sharing the same $\psi$. From this Alexander concluded that if a function

$w=a_{1} z+a_{2} z^{2} / 2+\cdots+a_{n} z^{n} / n$ has $\left\{a_{k}\right\}$ a nonincreasing sequence of positive numbers, then it satisfies the stated conditions and is therefore univalent. This follows since $w$ can be written as $w=a_{n} w_{n}+\left(a_{n-1}-a_{n}\right) w_{n-1}+\cdots+\left(a_{1}-a_{2}\right) w_{1}$ where $w_{n}=z+z^{2} / 2+\cdots+z^{n} / n$, each of which uses $\psi=\arg (z /(1-z))$. A similar theorem was proved for $w=a_{1} z+a_{3} z^{3} / 3+\cdots+a_{2 k+1} z^{2 k+1} /(2 k+1)$ where the $\left\{a_{k}\right\}$ is again nonincreasing and positive. The result for infinite power series of the same form and same condition on the coefficients then follows by a limiting argument.

The geometric aspect of this section appears to have been forgotten by subsequent authors, although his result on nonincreasing coefficients received attention. A later work which has some bearing on the mapping aspect of the section is that of Paatero [107] in 1931 in which the author defined the class of functions now known as bounded boundary rotation: a function is said to be of bounded boundary rotation with constant $K$ if the total absolute turning of the tangent vector to the image of the unit circle is less than or equal to $K \pi$. (If there is no boundary function the turning is defined by taking the limit as $r$ approaches 1 of the turning of the tangent vector to the image of the circle of radius, $r, 0<r<1$.) Although first introduced by Loewner in [83], Paatero was the first to develop properties of the class in detail. In $\S 7$ of his work he proved that if the total tangent turning of a function is less than $4 \pi$ then the function is univalent; the argument is very geometric and has much in common with Alexander's, though Alexander's work is not referenced. (See appendix $[\mathbf{A 5}]$.)

\section{Sections of Power Series, Monotonicity, and Convolutions Revisited}

Alexander's results in $\S 8$ were bound up in two lines of research which were very active from the early years of the twentieth century through the 1930's. We will describe the role of his contributions in this section.

In 1928 Gabor Szegö published a paper [144] in which he proved that every power series univalent in the unit disc has all its partial sums, or "sections", univalent in $|z|<1 / 4$, but not necessarily in any larger disc; he also proved the same theorem with "univalent" replaced by "starlike" for series of the form $z+a_{2} z^{2}+\ldots$ This paper stood in the tradition of research on the extent to which sections of power 
series or Fourier series share the properties of the entire series. This research goes back to Lebesgue's studies in the early 1900's of uniform bounds for sections of Fourier series of a function of bound one. Among the properties which a given power series possessed and which its sections would be expected to display, perhaps on a disc of smaller radius, were: having bound $M$ in the unit disc, convexity, starlikeness, vertical convexity, and having positive real part. Among the contributors to this literature were Fejér, E. Landau, Robertson, Carathéodory and O. Toeplitz, and many other lesser known mathematicans. A summary of the results up to 1936 was given by Szegö in an address to the American Mathematical Society in St. Louis on January 1 of that year [146].

The Alexander-Noshiro-Kobori condition on a power series is easily seen to be a condition on an analytic function which guarantees that its sections, which must then also satisfy the same condition, are univalent, starlike, and of bounded turning on the entire unit disc. The inheritance of univalence was pointed out by Itihara [61] in a 1929 paper which also provided generalizations of the coefficient condition. The inheritance of the starlike property was noted by Noshiro and the analogous result for convexity by Kobori in their previously mentioned papers. The coefficient condition considered in $\S 8$ of Alexander's paper received more attention: in 1935 [121] Robertson noted that although Szegö remarked that his radius of $1 / 4$ could not be improved "In some cases, however, one can say that the partial sums are univalent in the whole circle. J. W. Alexander has shown, for example, that if the coefficients of $f(z)=z+\sum_{n=2}^{\infty} a_{n} z^{n}$ are real and positive and such that the numbers $n a_{n}$ form a decreasing sequence, then not only $f(z)$ but all its partial sums are univalent in the unit circle" [p. 241]. He went on to prove that if all the partial sums of a univalent function are themselves univalent, then so are all the first Cesàro means of these partial sums. His proof uses the Dieudonné criterion and a result on zeroes of polynomials due to Obreschkoff.

The idea of using Cesàro means to "improve" the properties of the partial sums of a power or Fourier series goes back to Fejér's classical theorem on the first arithmetic means of the Fourier series of a continuous function, and Fejér also proved a theorem on the effect of averaging the sums of a vertically convex function: in 1933 he proved [38] that the third arithmetic means of a vertically convex function are themselves vertically convex in the entire disc. This was his way of dealing with the kind of questions posed by Szegö's 1928 paper, to which he referred on several 
occasions. This result was generalized by Robertson in 1950 [123] by showing that the convolution of any two vertically convex functions remains vertically convex; choosing one of the two to be the third arithmetic mean of $z /(1-z)$ (shown by Egerváry to be convex [34]), we get Fejér's theorem. This theorem provided another route by which convolutions became of interest in geometric function theory, and questions about the closure of classes of univalent functions under convolution became widespread, especially after the publication of Pólya and Schoenberg's paper [109] in 1958, which mentions Fejér's work in its introduction. This paper posed an equivalent problem for convex functions: is the Hadamard product of two univalent convex functions univalent and convex? The answer in the affirmative was given by Ruscheweyh and Sheil-Small in 1973 [126].

The other line of thought into which Alexander's theorems of this section entered was that involving properties of power series whose coefficients are positive and satisfy a monotonicity condition of some sort. Perhaps the first theorem of this sort was proved by G. Eneström in 1893 [35], in a paper entitled "Derivation of a General Formula for the Number of Pensioners in a Closed Pension Fund at a Given Time", a follow-up to an earlier paper "Use of a Method from Population Statistics for Solving a Problem in Pension Funds." In these papers Eneström was trying to develop a mathematical model for the number of civil servants in government pension funds. In this unlikely setting he proved a lemma: if $a_{0}+a_{1} z+a_{2} z^{2}+\cdots+a_{n} z^{n}$ has $a_{0} \geq a_{1} \geq \cdots \geq a_{n-1} \geq a_{n}>0$, then all the roots of the polynomial have absolute value greater than or equal to one. The theorem was rediscovered by S. Kakeya in 1912 [67], and thus goes under the name Eneström-Kakeya theorem. Many mathematicians, Hurwitz among them, contributed to generalizations and refinements of this result, and it was used by Landau in his 1916 book [79, p. 26] as a lemma in his derivation of the bounds of the partial sums of a function of bound one in the unit disc. If this lemma is applied to the derivative of a polynomial, we see that if $f(z)=z+a_{2} z^{2}+\cdots+a_{n} z^{n}$ satisfies $1 \geq 2 a_{2} \geq 3 a_{3} \geq \cdots \geq n a_{n}$ then it must be locally univalent. Alexander's theorem of $\S 8$ thus is an improvement of this local result to global univalence, and his example $z+z^{2} / 2+z^{3} / 3+\cdots+z^{n} / n$ shows that it is best possible in terms of location of critical points.

Fejér again made a contribution with his classical theorem that a sine series with positive coefficients which are doubly monotone is positive over the interval $[0, \pi]$ 
[41] (The idea of non-negativity of cosine series through double monotonicity of coefficients goes back to W. H. Young in 1913 [159], in a theorem rediscovered by Fejér in 1925 [37].) When applied to power series this result implies that a power series with doubly monotone positive coefficients is typically real. Thus a natural line of investigation is to see what kinds of monotonicity conditions imply univalence. Alexander's appears to have been the first such condition. In a later paper [40] Fejér quoted Alexander's result when he proved his follow-up: a power series in which the coefficients are positive and satisfy the condition that $n a_{n}$ are doubly monotone is starlike. Robertson in 1945 [122] added that such functions are vertically convex as well. Again this area proved very active in the 1930's. In [41], for example, Fejér again produced a result of this nature in which a power series whose coefficients exhibited four-fold monotonicity must represent a vertically convex univalent function. In a 1969 paper [87] T. H. MacGregor reiterated Alexander's contribution to monotonicity criteria, noted that "The idea of of a close-to-convex function is quite suggested by Alexander's paper", and proved more theorems on the monotonicity theme. He also acknowledged prior work from 1967 [116] on the same criteria. Other results both old and new linking monotonicity conditions and geometric properties of univalent functions are given in [46], [87], and [132].

\section{Some Topological Considerations}

Although this essay has been concerned with the ramifications of Alexander's paper in the field of univalent function theory, considering his later prominence as a topologist it is worth noting that questions of a purely topological nature arise from his work as well.

In [70] Kaplan offered possible generalizations of his results on close-to-convex functions to mappings of the complex plane with positive Jacobian. He reported that his colleague C. J. Titus conjectured that if a closed curve (not necessarily arising from the boundary values of an analytic mapping) has (1) a tangent which never turns back through an angle greater than $\pi$, (2) a tangent winding number equal to $2 \pi$ and (3) a non-negative winding number around every point in the plane, then the curve is a simple closed curve. Kaplan's work shows that if $f$ is analytic on the unit disc and (1) holds on every circle $|z|=r<1$, and if $f^{\prime}(z) \neq 0$ in the disc, then the image of each circle $|z|=r<1$ is a simple closed curve. The nonvanishing of $f^{\prime}(z)$ in the unit disc can be transformed into condition (2) for a general curve, and analyticity can be replaced by condition (3). Thus a natural extension of Kaplan's 
result can be conjectured. In a 1959 paper [148] Titus succeeded in proving that a closed "normal" curve is simple and positively oriented if and only if the tangent winding number is not greater than one, the tangent winding number of each subloop is nonnegative, and the index of the image of the curve around a point in the plane is nonnegative. The proof involves the theory of normal curves as introduced by Whitney in 1937.

In a later paper [115] J. R. Quine proved the conjecture as stated above using topological degree theory. In his introduction he mentioned Alexander's early work as well as Kaplan's introduction of close-to-convex functions. He obtained Kaplan's theorem as a corollary of his result. Quine had earlier [114] proved another conjecture of Titus [149] that, with certain trivial exceptions, a polynomial of degree $n$ takes the unit circle into a curve which has at most $(n-1)^{2}$ self intersections. The basic tool used in the proofs is Bézout's theorem, a result in the theory of algebraic curves. The bound is attained for functions of the form $p(z)=z+z^{n} / \epsilon$, for $\epsilon$ sufficiently small. This paper can be seen as the working out of the self-intersection topic from Alexander's paper, where conditions were imposed to assure the complete lack of self-intersection of the image curve of the unit circle under a polynomial.

We have stressed all along that Alexander's paper appeared at a time of transition from arguments based on geometric appeals to analytically rigorous treatments of subject matter, and an excellent illustration of this trend can be found in the proofs of the Jordan Curve Theorem given in Alexander's day. Jordan's original proof was given in 1893, but was considered incomplete due to its assumption of the validity of the theorem for closed polygons. Other proofs were given in subsequent years including one by Alexander himself [27, refs: 8, 12], published in 1920 but read before the American Mathematical Society in July 1916, a year after the appearance of his univalent function paper. His proof was based on chains, or systems of polygons, and their combinatorial properties, and was capable of generalization to higher dimensions. Two years prior to this G. N. Watson published [154], whose avowed purpose was to put on a rigorous basis the propositions necessary for a proof of Cauchy's Integral Theorem, among which was Jordan's Theorem. He followed the treatment of this subject given by earlier authors, and said in the introduction, "My endeavor has been to place the whole theory on a definitely arithmetical basis without appealing to geometrical intuitions" [Preface]. It appears that the project 
of firming up the topological foundations of the theory of boundary curves was well under way at this time.

Another example of this tendency, also relevant to Alexander's work, was given by Watson in 1916 [155, pp. 227-242]. He considered Jordan's Theorem in the following way: if a simple closed curve is given, $Q$ a fixed point not on the curve, and $P$ a variable point on it, then the angle between $Q P$ and the $x$-axis changes by zero or $2 \pi$ as $P$ describes the curve. He then examined an analogous question, that of the turn of the tangent vector to the curve, and proved that the angle between the tangent vector and the $x$-axis changes by $2 \pi$ as $P$ describes the curve once. This theorem is of interest in Alexander's section on close-to-convex functions, and would most likely be considered "obvious", to quote Whittaker and Watson in their classic book on Analysis [156], but Watson's proof runs fifteen or so pages in length.

As a final example, let us consider the theorem for which Watson's lemma mentioned above was undertaken. In 1898 H. M. MacDonald [84] investigated the zeroes of the Bessel functions, and in doing so proved the following theorem: the number of zeroes of an analytic funtion $f(z)$ in the region bounded by a contour on which $|f(z)|=m$ is one more than the number of critical points of $f$ in the same region. His argument was quite geometrical: he asserted that if $m$ is small, then the set $\{z:|f(z)|=m\}$ consists of $N$ closed curves surrounding the $N$ zeroes of $f$, and "... as $m$ increases, these ovals increase until two of them coalesce, the point at which they coalesce being a double point on the curve corresponding to that particular value of $m$. When $m$ has increased to its final value ... the $N$ closed curves have coalesced into one closed curve, and therefore $N-1$ double points have been passed through" [pp. 576-577]. He then argued that these double points are zeroes of $f^{\prime}$, and treated separately the case in which zeroes may coincide. By contrast Watson's proof of this theorem, found in Whittaker and Watson [p. 121], involves calculating the difference in the winding number integrals for $f$ and $f^{\prime}$, an expression which, after some computation, is found to be the change in $\psi / 2 \pi$ (where $\psi$ is the angle that the tangent to the curve bounding the region makes with the positive $x$-axis) as the curve is traversed once in the counterclockwise direction. Due to Watson's lemma, this number is one. Very little is thus left to geometric intuition.

MacDonald's theorem has an interesting history which we will abbreviate here. It was generalized by Denjoy in 1918 [28] to the case in which the number of distinct zeroes and poles in the region total $n$, and the conclusion being that the derivative 
has $n-1$ zeroes distinct from the zeroes and poles of $f$. The hypothesis $|f(z)|=m$ on the boundary replaced by "the argument of $f$ varies in a constant sense when $z$ describes $C$ [the boundary of the region on which $f$ is defined]" [p. 31]. (Note the similarity to Alexander's original definition of starlike mappings.) This result can in a sense be considered a generalization of Alexander's assertion of the univalence of starlike mappings by revealing the relation between zeroes and critical points for a non-univalent starlike mapping. In 1927, apparently unaware of Denjoy's work, M. Ålander [1] generalized MacDonald's result in the following way: if $C$ encloses $p$ zeroes of $f$ and $q$ poles, where the poles are distributed in $b$ distinct points, then it encloses $p+b-1$ critical points of $f$. Ålander's work in turn was considered in a purely topological light by S. Stoïlow in 1930 [134], who invoked his notion of an interior mapping: an interior mapping is any function which is an open mapping such that the preimage of any point is a totally disconnected set. Stoïlow's paper generalized Ålander's work by replacing the number of zeroes and poles by the "degree" of the interior mapping, properly defined, and critical points by "points of ramification", also suitably defined. This formula was noted by Alexander's colleague at the Institute for Advanced Study, M. Morse, in a set of lectures given at Princeton in 1945 [96, p. 79]. He saw it as a precursor to his "general order theorem" for interior transformations. In such a fashion a humble theorem on complex polynomials found interesting generalizations in the realm of topology.

\section{Conclusion}

One wonders whether Alexander himself was aware of these developments, and indeed whether he ever thought again of his youthful effort in univalent function theory. His subsequent publications show no trace of this interest, and his 1915 paper remains his lone contribution to complex analysis. We hope the reader now shares our opinion of its unique place in that literature and has come to appreciate its innovative character and wellspring of ideas.

\section{APPENDIX}

A1: Our convolution story comes full circle as we highlight a modern farreaching generalization of Alexander and Kakeya's results on critical points achieved by Volker Kasten [74] in 1986. For $P_{n}=\{f: f(z)=1+$ $a_{2} z^{2}+a_{3} z^{3}+\cdots+a_{n} z^{n}, f(z) \neq 0$ in $\left.|z|<1\right\}$ we let $m(p)=\min \{|z|$ : 
$\left.p^{\prime}(z)=0, p \in z P_{n-1}\right\}$, and consider polynomials of the type $T(r, n)(z)=$ $\left((z+r)^{n}-r^{n}\right) / n r^{n-1} \in z P_{n-1}$, these are clearly the extremals considered by Alexander. Then to each $p \in z P_{n-1}$ we assign the test polynomial $T(p)=T(m(p), n)$. Kasten stated Alexander's result on convex polynomials together with Kakeya's Theorem as Theorem AK: for all $p \in z P_{n-1}$ we have that convexity of $T(p)$ implies convexity of $p$, and univalence of $T(p)$ implies univalence of $p$. His paper generalizes theorem AK as follows: a subset of $B$ of $z P_{n-1}$ is said to have the test property if, for all $p \in z P_{n-1}$, $T(p) \in B$ implies $p \in B$. The convex univalent and univalent polynomials have this property by Theorem AK. Kasten's theorem is that any subset $B$ of $z P_{n-1}$ satisfying a Dieudonné-type criterion has the test property, and so the extremal value for $m(p)$ over such a class can be determined by looking at the polynomials $T(r, n)$. The proof of this theorem relies on many of the results on convolution material obtained by Kasten and Ruscheweyh in the 1980's in the abstract setting of "duals" of subsets of the collection of normalized analytic functions. As corollaries he obtained the test property for the classes of polynomials which are starlike or convex of order $\alpha$, as well as those of bounded turning, thus producing Alexander's result in $\S 5$. It is interesting to see how convolution theory as developed in the abstract was in turn applied to the types of problems which contributed to the rise of convolution methods initially. It hardly needs to be emphasized, however, that these convolution methods are quite removed from Alexander's geometric intuition.

A2: We shall call the class of functions in the unit disc $\left\{w: \Re\left(e^{i a} w^{\prime}\right)>0\right\}, a$ real constant, the class of functions of bounded turning. This class seems not to have received the immediate attention that the convex and starlike sets did. It appears that no reference to this class was made from 1915 to 1934, when three authors mentioned the defining condition. Julius Wolff [158] gave a detailed geometric proof of the univalence of functions of positive real part defined in a half plane. He began by noting that if $F=U+i V$ has $\Re\left(F^{\prime}\right)>0$ then any curve $G_{c}=\{z: V(z)=c, c$ constant $\}$ can cut a line parallel to the imaginary axis in one and only one point, and continued to argue by cases that there are no other curves $G_{c}^{\prime}$ to the left or right of $G_{c}$ 
and disjoint from it. He concluded that $G_{c}$ is unique, hence since on each such curve $U$ takes a value only once, it follows that $F$ is univalent.

The theorem that a function on a convex domain whose derivative has positive real part is univalent is usually referred to as the Noshiro-Warshawski Theorem, since in 1935 these two authors published papers with essentially the same straightforward analytical proof of this result [100], [153]. Following Warshawski, for example, we let $f^{\prime}=u+i v$, then for $z_{1} \neq z_{2}$ we have $f\left(z_{2}\right)-f\left(z_{1}\right)=\int_{z_{1}}^{z_{2}} f^{\prime}(s) d s$ where the integral is taken along the straight line path from $z_{1}$ to $z_{2}$, call it $s=z_{1}+t e^{i a}, 0 \leq t \leq L$. Then $\left.\left|f\left(z_{2}\right)-f\left(z_{1}\right)\right|=\mid \int_{t=0}^{L} f^{\prime}(s) e^{i a} d t\right)|=| \int_{0}^{L} f^{\prime}(s) d t|\geq| \int_{0}^{L} u(s) d t \mid>0$. In these proofs no geometric conclusion is possible about the shape of the mapping curve, but in Noshiro's paper there is a note at the bottom of page 151 which reads: "When I read this paper at the annual meeting of the Physico-Math. Soc. of Japan, held in April of 1934, Prof. Kakeya kindly remarked to me that this theorem can easily be proved by a geometrical consideration". In any event, the focus in each paper was not on this result, which was used only as a lemma, but the idea of the fundamental theorem of calculus proved useful in later investigations in which the issue was the type of domain on which $\Re\left(f^{\prime}(z)\right)>0$ assured univalence of $f$.

Interest in the bounded turning class as such was renewed in the 1950's when Zmorovich published a series of papers investigating the usual growth and coefficient questions; see [45] for a summary of these. Zmorovich introduced the terminology "bounded turning". In 1961 T. H. MacGregor published part of his Ph. D. thesis as [85] "Functions Whose Derivative has a Positive Real Part" and treated many of the same questions. This work seems also to be the first to acknowledge Alexander's pioneering work on the class.

A3: The examples of Osgood and Fredholm, which invoked the Alexander condition to prove univalence, can be improved by the Alexander-NoshiroKobori starlikeness/convexity result, as was pointed out by Goodman [47] in 1957, and later L. Zalcman [160] in 1974. Combining their comments one can produce an example of a univalent power series convergent in the unit circle which is $C^{\infty}$ on the boundary, having the unit circle as a natural boundary, and mapping onto a convex domain. Such a mapping thus produces a convex, 
$C^{\infty}$ curve which must then be non-analytic on any subarc, otherwise the Schwarz reflection principle would provide an analytic continuation across the natural boundary. The example is given by $f(z)=z+\sum_{k=N}^{\infty} z^{2^{k}} / k$ ! The gap condition on the powers ensures that the unit circle is the natural boundary by the Hadamard Gap Theorem, and if $N$ is chosen sufficiently large, we have by Alexander-Noshiro-Kobori that the mapping is convex. $C^{\infty}$ on the boundary is ensured by absolute convergence of all derivatives on the boundary. Perhaps the existence of this topological construct would have been of interest to Alexander.

A4: A second geometric touch was added by T. J. Suffridge, who among modern analysts has produced work closest to Alexander's in style and content. In 1974 [140] he proved a theorem which sharpened Alexander's results and characterized polynomials of degree $n$ whose critical points lie on the unit circle at a greater angle than the separation considered by Alexander, $2 \pi /(n+1)$. We will conclude this section by describing this contribution. If we consider the course of the tangent vector $T$ to the image of the unit circle under a polynomial mapping with critical points on the boundary, the argument of $T$ will decrease by $\pi$ as it passes through a critical point, and increase in argument between two critical points. If the argument change between two critical points is large enough, the net change in $\arg (T)$ as $T$ passes through two successive critical points is $-2 \pi+$ (argument change between critical points) $>-\pi$. It is plausible that the requirement that $\Delta \arg (T)>-\pi$ as $T$ varies between any two points on the image circle will ensure univalence; an extremal case occurs at a critical point itself. This condition on a mapping was considered by Alexander in $\S 8$ (presumably motivated by these considerations in $\S 7$ ) and applied to $f_{1}, f_{2}$ and $f_{3}$.

Suffridge proved that for polynomials of the form $P(z)=z+a_{2} z^{2}+\cdots+$ $\eta z^{n} / n,|\eta|=1, P$ satisfies $\Delta \arg (T)>-\pi$ for $T$ the tangent vector to the image of $|z|=r, r<1$, as $T$ varies between any two points on the image of $|z|=r$ if and only if all critical points of $P$ are distinct, lie on $|z|=1$, and are separated in argument by $2 \pi /(n+1)$. The set [138] through [142] contains much work of this nature. The tangent vector condition discussed by Suffridge is treated in more detail in the next section. 
A5: In the 1935 paper of Ozaki [106] there is work which relates to the closeto-convex class, because the author proved the following theorem: supposed that $g$ is a convex function in the unit disc, and suppose $f$ is analytic in the unit disc, $\alpha$ real and $\Re\left[e^{i \alpha} f^{\prime}(z) / g^{\prime}(z)\right]>0$ in the unit disc, then $f$ is univalent there. The connection to Alexander's work is obvious: if $\alpha=0$ then Ozaki's condition can be rewritten as $\Re\left[z f^{\prime}(z) / z g^{\prime}(z)\right]>0$ which is equivalent to $-\pi / 2<\arg \left(z f^{\prime}(z)\right)-\arg \left(z g^{\prime}(z)\right)<\pi / 2$, so Alexander's criteria are satisfied with $\psi=\arg \left(z g^{\prime}(z)\right)=$ normal to image of unit circle under $g$, an increasing function of $\arg (z)$ since $g$ is convex. (This again assumes that $f$ and $g$ are defined on the unit circle.) The proof is a simple change of variables: setting $\phi=f\left(g^{-1}(z)\right)$, then $f^{\prime}(z)=\phi^{\prime}(g(z)) g^{\prime}(z)$. Thus $\Re\left[f^{\prime}(z) / g^{\prime}(z)\right]=$ $\Re\left[\phi^{\prime}(g(z))\right]>0$ in the image of the unit circle under $g$. Thus by the criteria of $\S 6$ of Alexander, cited as Noshiro's result by Ozaki, the proof is complete.

Ozaki did not cite Alexander's work on this material, or stress any geometric interpretation of his theorem, but arrived at the same results. $\mathrm{He}$ first replaced $g^{\prime}$ by $h$ starlike and restated the theorem with $h$; interestingly here he did cite Alexander's result allowing this replacement. He then chose $h$ to be $z /(1-z), z /(1-z)^{2}$, and $z /\left(1-z^{2}\right)$ and got many corollaries among which are Alexander's two theorems on monotone decreasing coefficients. For example, choosing $g(z)=\log (1 /(1-z))$ and $f(z)=\sum_{n=1}^{\infty} a_{n} z^{n}$ gives $\Re\left[f^{\prime}(z) / g^{\prime}(z)\right]=\Re\left[(1-z) f^{\prime}(z)\right]=1-\Re\left[\sum_{n=1}^{\infty}\left(n a_{n}-(n+1) a_{n+1}\right) z^{n+1}\right]>$ $1-\sum_{n=1}^{\infty}\left|n a_{n}-(n+1) a_{n+1}\right|$; thus if the sum is less than 1 we have $f$ univalent. We then have that if $\sum_{n=1}^{\infty}\left|n a_{n}-(n+1) a_{n+1}\right|<1$ then $f$ is univalent. He obtained Alexander's result as a corollary in the form $f(z)=z+a_{2} z^{2}+\cdots$ with $1 \geq 2 a_{2} \geq 3 a_{3} \geq \cdots \geq 0$ implies $f$ is univalent in the unit disc. We may note that in Alexander's work the ray which stays close to the normal vector of the given function and guarantees its univalence need not a priori come from the normal to some other convex function; in $f_{2}$ this is the case, but in $f_{3}$ his chosen ray cannot be a normal of this sort. Again, the geometric nature of Alexander's arguments stand in contrast to the analytic approach in Ozaki's paper.

The decisive work which connected the above papers was Kaplan's 1952 "Close-to-Convex Schlicht Functions" [70]. After having outlined in [69] Wolff's proof that a function of positive real part in a half plane is univalent, 
he sought to generalize this and arrived at the notion of close-to-convex functions, defined as in Ozaki's theorem with $\alpha=0$ [73]. The name of the class is motivated by the geometric interpretation noted by Alexander, that the normal of $f$ never differs from the normal of $g$ by more than $\pi / 2$, though Kaplan was unaware of Alexander's work [73]. He proved that close-to-convex functions are univalent as Ozaki did, then cited many subclasses, including the starlike mappings (by Alexander's Theorem). But his main contribution was to prove that a necessary and sufficient condition for a function with non-vanishing derivative to be close-to-convex (hence univalent) is that for all $r<1$ we have that the decrease in argument of the tangent vector to the image curve of $|z|=r$ is never less than $\pi$. This theorem eliminates the problems with the possible non-existence of the function on the unit circle and also shows that the ray in Alexander's theorem can always be chosen as the normal to some associated convex function. Kaplan noted the geometric interpretation of the class.

There is the following anecdote concerning this class [20]: When C. Pommerenke was writing his book [111] on univalent functions, he included a section on close-to-convex functions defined as Ozaki had done. When it was pointed out to him that Alexander had dealt with this class, Pommerenke said that this showed how cunning Alexander must have been to characterize the class before it had even been defined! This story also shows the modern tendency to think of a class as being defined analytically and "characterized" geometrically.

\section{REFERENCES}

[1] Ålander, M. Sur une propriété des fonctions méromorphes à l'intérieur d'une ligne de module constant, C. R. Acad. Sci. Paris 184(1927) 1411-1413.

[2] Alexander, J. W. II, Functions which map the interior of the unit disk upon simple regions, Ann. of Math. 17(1915) 12-22.

[3] Askey, R. and Steinig, J., A monotonic trigonometric sum, Amer. J. Math. 98, No 2 (1976) 357-365.

[4] Askey, R., Orthogonal polynomials and special functions, SIAM Press, Philadelphia (1975) vol. 21.

[5] Askey, R., letter to the authors.

[6] Başgöze, T., On the radius of univalence of a polynomial, Math. Z. 105(1968) 299-300. 
[7] Başgöze, T., On the univalence of certain classes of analytic functions, J. London Math. Soc. (2) 1(1969), 140-144.

[8] Başgöze, T., On the univalence of polynomials, Compositio Mathematica 22(1970) 245-252.

[9] Bellman, R. and Kalha, R., Selected Papers on Mathematical Control Theory, Dover Publications, 1964.

[10] Bernardi, S. B., Bibliography of Schlicht Functions, Polygonal Publishing House, Washington, N. J., 1982.

[11] Bieberbach, L., Aufstellung und Beweis des Drehungssatzes für Schlichte Konforme Abbildungen, Math. Z. 4(1919) 295-305.

[12] Bieberbach, L., Eine hinreichende Bedingung für schlichte Abbildungen des Einheits- Kreises, Jour. für Math. 157(1927) 189-192.

[13] Bieberbach, L., Vorlesungen Über Algebra, Verlag und Druck von B. G. Teubner, Berlin, 1928.

[14] Bieberbach, L., Lehrbuch der Funktionentheorie, Bd 1, 2, B. G. Teubner, Leipzig/Berlin, 1930.

[15] Biernacki, M., Sur les équations algébriques contenant des paramètres arbitraires, Thèse, Bulletin de l'Académie polnaise des Sciences et des Lettres A(1927) 546-685.

[16] Biernacki, M., Sur l'integrale des fonctions univalentes, Bull. Acad. Polon. Sci. Ser. Sci. Math. Astr. Phys. 8(1960) 29-34.

[17] Bojancu, B. D., Rahman, Q. I. Szynal, J., On a conjecture of Sendov about the critical points of a polynomial, Math. Z. 190(1985) 281-285.

[18] Brannan, D. A., On functions of bounded boundary rotation I, Proc. Edinburgh Math. Soc. 16(1968-1969) 339-347.

[19] Brannan, D. A., On univalent polynomials, Glasgow Math. J. 11(1970) 102-107.

[20] Brannan, D. A., letter to the authors.

[21] Brannan, D. A., Brickman, L., Coefficient regions for starlike polynomials, Ann. Univ. Mariae Curie-Skłodowska 29, A(1975) 15-21.

[22] Bustoz, J., Jacobi polynomial sums and univalent Cesàro means, Proc. Amer. Math. Soc. $\mathbf{5 0}(1975)$ 259-264

[23] Čakalov, L. N., On domains of univalence of certain classes of analytic functions, Soviet Math. Doklady vol. 1, No. 3(1960) 781-783.

[24] Carathéodory, C., Über den Variabilitätsbereich der Koeffizienten von Potenzreihen, die Gegebene Werte nicht annehemen, Math. Ann. 64(1907) 95-115.

[25] Carslaw, H. S., An introduction to the theory of Fourier's series and integrals, Third Revised Edition, Dover Press, 1990.

[26] Clunie, J. G., Keogh, F. R., On starlike and convex schlicht functions, J. London Math. Soc. 35(1960) 229-233.

[27] Cohen, L., James Wadell Alexander 1888 - 1971, Bull. Amer. Math. Soc . 79, No.(5)(1973) 900-903.

[28] Denjoy, A., Sur une propriété générale des fonctions analytiques, C. R. Acad. Sci. Paris 166(1918) 31-32.

[29] Dieudonné, J., Recherches sur quelques problèmes relatifs aux polynomes et aux fonctions bornées d'une variable complexe, Ann. École Norm. Sup. 48(1931) 247-358. 
[30] Dieudonné, J., Sur quelques propriétés des polynomes, Actualités Scientifiques et Industrielles, Exposés Mathématiques 114(1934), Herman \& Cie, Paris.

[31] Dieudonné, J., La Theorie Analytique des Polynomes d'une Variable, Gauthier-Villars, Paris, 1938.

[32] Duren, P. L., Univalent Functions, Springer Verlag, New York, 1983.

[33] Egerváry, E., On a maximum-minimum problem and its connexion with the roots of equations, Acta. Univ. Hung. Francisco-Josephinae 1(1922) 39-45.

[34] Egerváry, E., Abbildungseigenschaften der arithmetischen Mittel der geometrischen Reihe, Math. Z. 42(1937) 221-230.

[35] Eneström, G., Härledning af en allmän formel för antalet pensionärer, som vid en godtycklig tidpunkt förefinnas inom en sluten pensionskassa, Öfversigt af Kongl. Vetenskaps-Akademiens Förhandlingar (Stockholm) 50(1893) 405-415.

[36] Fejér, L., Sur les singularités de la séries de Fourier des fonctions continues, Ann. Ecole Normale Supérieure 28(1911) 64-103.

[37] Fejér, L., Über die Positivität von Summen, die nach trigonometrischen oder Legendreschen Funktionen fortschreiten (erste Mitt.), Acta Litt. ac Scient. Szeged 2(1925) 75-86

[38] Fejér, L., Neue Eigenschaften der Mittelwerte bei den Fourierreihen, J. London Math. Soc. 8(1933) 53-62.

[39] Fejér, L., G. Szegö, On new properties of the arithmetic means of partial sums of Fourier series, Journal of Math. and Physics 13(1934) 1-17.

[40] Fejér, L., Untersuchungen über Potenzreihen mit Mehrfach Monotoner Koeffizientenfolge, Acta Litt. ac Scient. Szeged 8(1936) 89-115.

[41] Fejér, L., Trigonometrische Reihen und Potenzreihen mit mehrfach monotoner Koeffizientenfolge, Trans. Amer. Math. Soc. 39(1936) 18-59.

[42] Fejér, L., G. Szegö, Special conformal mappings, Duke Math. J. 18(1951) 535-548.

[43] Fournier, R., On integrals of bounded analytic functions in the unit disc, Complex Variables Theory Appl. 11(1989) 125- 133.

[44] Fuller, A. T. ed., Stability of Motion, Halstead Press, New York, 1975.

[45] Galperin, I. M., The theory of univalent functions with bounded rotation, Izv. Vyss. Ucebn. Zaved. Mathematika, No. 3 4(1958), 50-61.

[46] Gluchoff, A., Hartmann, F., Univalent polynomials and non-negative trigonometric sums, Amer. Math. Monthly, No. 6 105(1998) 508- 522.

[47] Goodman, A. W., Univalent functions and non-analytic curves, Proc. Amer. Math. Soc. 8(1957) 598-601.

[48] Goodman, A. W., Univalent Functions, Vols. 1 \& 2, Polygonal Publishing House, Washington, New Jersey, 1983.

[49] Goodman, A. W., Uniformly starlike functions, J. Math. Anal. Appl., 155(1991) 364-370.

[50] Goodman, A. W., Uniformly convex functions, Ann. Polon. Math., 56(1991) 87- 92.

[51] Grace, J. H., The zeroes of a polynomial, Proc. Camb. Phil. Soc. 11(1902) 352-397.

[52] Gronwall, T. H., Über die Gibbssche Erscheinung und die Trigonometrischen Summen $\sin x+$ $1 / 2 \sin 2 x+\cdots+1 / n \sin n x$, Math. Ann. 72(1912) 228-243. 
[53] Gronwall, T. H., Some remarks on conformal representation, Ann. of Math. (2) 16(1914-1915) $72-76$.

[54] Gronwall, T. H., Sur la déformation dans la représentation conforme, C. R. Acad. Sci. Paris 162(1916) 249-252.

[55] Gronwall, T. H., Sur la déformation dans la représentation conforme sous des conditions restrictives, C. R. Acad. Sci. Paris 162(1916) 316-318.

[56] Gronwall, T. H., On the power series for $\log (1+z)$, Ann. of Math., Series 2, No. 2 18(1916) 70-73.

[57] Hardy, G. H., Rogosinski, W. W., Fourier Series, Cambridge University Press, New York, 1944.

[58] Heawood, P. J. Geometrical relations between the roots of $f(x)=0, f^{\prime}(x)=0$, Quart. J. Math. 38(1907) 84-107.

[59] Hibbert, L., Univalence et automorphie pour les polynomes et les fonctions entières, Bull. Soc. Math. France 66(1938) 81- 194.

[60] Hurwitz, A., Über die Bedingungen unter welchen eine Gleichung nur Wurzeln mit negativen reelen Theilen besitz, Math. Ann. 46(1895) 273-284.

[61] Itihara, T., Eine Bemerkung über die Schlichtheit der Potenzreihe und ihrer Abschnitte, Japanese Journal of Mathematics 6(1929) 183-187.

[62] Jackson, D., Über eine trigonometrische Summe, Rend. Circ. Mat. Palermo 32(1911) 257-262.

[63] Jahangiri, M., Silverman, H., Silvia, E. M., Inclusion relations between classes of functions defined by subordination, J. Math. Anal. Appl., No. 2 151(1990) 318-329.

[64] Jahrbuch über die Fortschritte der Mathematik, 45(1915-16) 672.

[65] James, I. M., letter to the authors, 1998.

[66] James, I. M., ed., History of Topology, North Holland, 1999.

[67] Kakeya, S., On the limits of roots of an algebraic equation with positive coefficients, Tohôku Math. J. 2(1912) 140-142.

[68] Kakeya, S., On the zeroes of a polynomial and its derivatives, Tohôku Math. J. 11(1917) 5-16.

[69] Kaplan, W., On Gross's star theorem, schlicht functions, logarithmic potentials, and Fourier series, Ann. Acad. Sci. Fennicae, Ser. A. I. Math.-Phys. 86(1951) 1-23.

[70] Kaplan, W., Close-to-convex schlicht functions, Mich. Math. J. 1(1952) 169-185.

[71] Kaplan, W., Convexity and the Schwarz-Christoffel mapping, Mich. Math. J. 40(1993) 217-227.

[72] Kaplan, W., On close-to-convex Schwarz-Christoffel mappings, Complex Variables Theory Appl. 33(1997) 137-143.

[73] Kaplan, W., letter to the authors, 1997.

[74] Kasten, V., Polynomial classes with a certain test property, Complex Variables Theory and Appl. 7(1986) 89-96.

[75] Klein, F., Funktionentheorie in geometrischer Behandlungsweise; Vorlesung gehalten in Leipzig 1880/81 Mit zwei Originalarbeiten von F. Klein aus dem Jahre 1882, BSB B. G. Teubner Verlagsgesellscaft, Leipzig, 1987, distributed by Springer-Verlag, Wien, New York.

[76] Kobori, A., Über sternige und konvexe Abbildungen, Mem. Coll. Sci. Kyoto A 15(1932) 267-278. 
[77] Koebe, P., Über die Uniformisierung beliebeger analytischer Kurven, Nach. Ges. Wiss. Gottingen (1907) 191-210.

[78] Koenig, J. F., On the zeroes of polynomials and the stability of linear systems, Jour. of Applied Physics 24(1953) 476-482.

[79] Landau, E., Darstellung und Bergründung einiger neuerer Ergebnisse der Funktionentheorie, Chelsea Publishing Co., New York, 1946

[80] Leftschetz, S., James Waddell Alexander (1888-1971), American Philosophical Society Yearbook (1973) 110-114.

[81] Lewandowski, Z., Quelques remarques sur les théorèmes de Schild relatifs à une classe de fonctions univalents, Ann. Univ. Mariae Curie-Skłodowska Lubin-Polonia 9, A(1955) 149155.

[82] Liénard, A., Chipart, M. H., Sur le signe de la partie réele des racines d'une équation algébraique, J. Math. Pures Appl. (6), 10(1914) 291-346.

[83] Loewner, K., Untersuchungen über die Verzerrung bei konformen Abbildungen des Einheitskreises, Leipzig Berichte 69(1917) 89-106.

[84] MacDonald, H. M., Zeroes of Bessel functions, Proc. London Math. Soc. 29(1898) 110-115.

[85] MacGregor, T. H., Functions whose derivative has positive real part, Trans. Amer. Math. Soc. 104(1962) 532-537.

[86] MacGregor, T. H., A class of univalent functions, Proc. Amer. Math. Soc. 15(1964) 311-317.

[87] MacGregor, T. H., Univalent power series whose coefficents have monotone properties, Math. Z. 112(1969) 222-228.

[88] Maclane, S., Topology and logic at Princeton, "A Century of Mathematics in America", ed. P. L. Duren, Amer. Math. Soc., Providence, R. I., 1989, pp. 217-221.

[89] Mansour, S., On extreme points in two classes of functions with univalent sequential limits, Ph. D. disseration, Univ. of Kentucky, 1972.

[90] Marden, M., Geometry of Polynomials, Amer. Math. Soc., Providence, R. I., 1966

[91] Maxwell, J. C., On Governors, Proc. Roy. Soc., 16(1868) 270-283.

[92] Merkes, E. P., Robertson, M. S., Scott, W. T., On products of starlike functions, Proc. Amer. Math. Soc. 13(1962) 960-964.

[93] Miller, S., Mocanu, P., Univalent integral operators, J. Math. Anal. Appl. (1)157(1991) 147165.

[94] Mittag-Leffler, G., Sur la représentation d'une branche uniforme d'une fonction monogène, I-V Acta Math. 23 43-62, 24 183-204 and 205-244, 26 353-391, 29 101-181 (1900-1905).

[95] Montel, P., Leçons sur les fonctions univalentes ou multivalentes, Gauthier-Villars, Paris, 1933.

[96] Morse, M., Topological Methods in the Theory of Functions of a Complex Variable, Princeton University Press, 1947.

[97] Mullender, P., On some conformal mappings, Simon Stevin 26(1949) 136-142.

[98] Nevanlinna, R., Über die Konforme Abbildung von Sterngebieten, Översikt av Finska Vetenskaps-Societetens Förhandlingar, No. 6 63(1920-1921) 1-21.

[99] Noshiro, K., On the star-shaped mapping of an analytic function, Proc. Imp. Acad. Japan 8(1932) 275-277. 
[100] Noshiro, K., On the theory of schlicht functions, J. Fac. Sci. Hokkaido Univ. Jap. 2(1934-35) 129-155.

[101] Obreschkoff, N., Sur les polynomes univalentes , C. R. Acad. Sci. Paris 198(1934) 2649-2650.

[102] Obreschkoff, N., Über algebraische Gleichungen, die nur Wurzeln mit negativen Realteilen besitzen, Math. Z. 45(1939) 747- 750.

[103] Osgood, W. F., Example of a single-valued function with a natural boundary, whose inverse is also single-valued, Bull. Amer. Math. Soc. 4(1898) 417-424.

[104] Osgood, W. F., Supplementary note on a single-valued function with a natural boundary, whose inverse is also single valued, Bull. Amer. Math. Soc. 5(1898) 17-18.

[105] Osgood, W. F., Lehrbuch der Funktionentheorie Verlag von B. G. Teubner, Leipzig, Vol I, $1923,4^{\text {th }}$ edition.

[106] Ozaki, S., On the theory of multivalent functions, Sci. Rep. Tokyo Bunrika Daigaku, Sec. A, 2(1935) 167-188.

[107] Paatero, V., Über die konforme Abbildung von Gebieten deren Ränder von beschränkter Drehung sind, Ann. Acad. Sci. Fenn., Ser. A 33(1931) 1-78.

[108] Pinchuk, B., A variational method for functions of bounded boundary rotation, Trans. Amer. Math. Soc. 138(1969) 107-113.

[109] Pólya, G., Schoenberg, I. J., Remarks on de la Vallée Poussin means and convex conformal maps of the circle, Pacific J. Math. 8(1958) 295- 334.

[110] Pólya, G. and Szegö, G., Problems and Theorems in Analysis, Vols. 1 \& 2, Springer-Verlag, 1976.

[111] Pommerenke, C., Univalent Functions, Vandenhoeck \& Ruprecht, Göttingen, 1975.

[112] Ponnusamy, S., Neighborhoods and Carathéodory functions, J. Anal. 4(1996) 41-51.

[113] Ponnusamy, S. and Vuorinen, M., Univalence and convexity properties for confluent hypergeometric functions Complex Variables Theory Appl. 36(1998) 73-97.

[114] Quine, J. R., On the self-intersections of the image of the unit circle under a polynomial mapping, Proc. Amer. Math. Soc., No. 1, 39(1973) 135- 140.

[115] Quine, J. R., Some topological theorems relating to close-to- convex functions, J. London Math. Soc., No. 2, 14(1976) 39-42.

[116] Reade, M. O., Umezawa, T., Some criteria for the multivalence of certain analytic functions, Colloq. Math. 16(1967) 23-26.

[117] Remak, R., Über winkeltreue und streckentreue Abbildung an einem Punkte und in der Ebene, Palermo Rend. 38(1914) 193-246.

[118] Remak, R., Über eine spezielle Klass schlichter Konformer Abbildungen des Einheitskrieses, Mathematica, Ser. B, 11(1943) 175-192.

[119] Robertson, M. S., A note on schlicht polynomials, Trans. Roy. Soc. Canada, No. 3, 26(1932) 43-48.

[120] Robertson, M. S., On the theory of univalent functions, Ann. Math. 37(1936), 374-408.

[121] Robertson, M. S., On the univalency of Cesàro sums of univalent functions, Bull. Amer. Math. Soc. 42(1936) 241-243. 
[122] Robertson, M. S., Univalent power series with multiply monotone sequences of coefficients, Ann. Math., No. 4, 46(1945) 533-555.

[123] Robertson, M.S., Applications of a lemma of Fejér to typically real functions, Proc. Amer. Math. Soc. 1(1950) 555-561.

[124] Robertson, M. S., Schlicht solutions of $W^{\prime \prime}+p W=0$, Trans. Amer. Math. Soc. 76(1954) 254-274.

[125] Rønning, F., A survey on uniformly convex and uniformly starlike functions, Ann. Univ. Mariae Curie-Skłodowska, Lubin- Polonia, No. 13, 47, A(1993) 123- 134.

[126] Ruscheweyh, S., Sheil-Small, T. B., Hadamard products of schlicht functions and the PólyaSchoenberg conjecture, Comment. Math. Helv. 48(1973) 119-135.

[127] Ruscheweyh, S., Neighborhoods of univalent functions, Proc. Amer. Math. Soc., No. 4, 81(1981) 521-527.

[128] Ruscheweyh, S., Convolutions in Geometric Function Theory, Les Presses de l'Université de Montréal, 1982.

[129] Schild, A., On a class of functions schlicht in the unit circle, Proc. Amer. Math. Soc. 5(1954) 115-120.

[130] Silverman, H., Complex Variables, Houghton Mifflin, Boston, 1975.

[131] Silverman, H., Univalent Functions with negative coefficients, Proc. Amer. Math. Soc. 51(1975) 109-115.

[132] Silverman, H., Silvia, E. M., Telage, N., Convolution conditions for convexity, starlikeness, and spiral-likeness, Math. Z. 162(1978) 125-130.

[133] Špaček, L., Contribution à la théorie des functions univalentes, Casopis Pest. Mat. 62(1932) 12-19.

[134] Stoïlow, S., Du caractère topologique d'une théorème sur les fonctions méromorphes, C. R. Acad. Sciences de Paris 190(1930) 251-253.

[135] Stoïlow, S., Leçons sur les principes topologiques de la théorie des fonctions analytiques, Gauthier-Villars, Paris, 1956.

[136] Strazdes, D. J., American Painting and Sculpture to 1945 in the Carnegie Museum of Art, Hudson Hills Press, New York, 1992, 43-51.

[137] Study, E., Konforme Abbildung Einfache-Zussammenhängender Bereiche, B. G. Teubner, Leipzig/Berlin, 1913.

[138] Suffridge, T. J., On univalent polynomials, J. London Math. Soc. 44(1969) 496-504.

[139] Suffridge, T. J., Extreme points in a class of polynomials having univalent sequential limits, Trans. Amer. Math. Soc. 63(1972) 225-237.

[140] Suffridge, T. J., Starlike functions as limits of polynomials, Advances in Complex Function Theory, Lecture Notes in Mathematics, Springer-Verlag, New York, 1974.

[141] Suffridge, T. J., Polynomials in Function Theory, "Contemporary Mathematics", Amer. Math. Soc. Press 38(1985) 31-85.

[142] Suffridge, T. J., Polynomial approximation of Schwarz-Christoffel mappings, unpublished manuscript delivered at AMS annual meeting, 1995. 
[143] Szegö, G., Bemerkungen zu einem Satz von J. H. Grace über die Wurzeln algebraischer Gleichungen, Math. Z. 13(1922) 28-56.

[144] Szegö, G., Zur Theorie der Schlichten Abbildungen, Math. Ann. 100(1928) 188-211.

[145] Szegö, G., Zwei Extremalaufgaben über Abbildungen, die durch Polynome vermittelt sind, Acta Lit. Ac. Scient. Univ. Hung. Franc. Jos. 5(1932) 237-245.

[146] Szegö, G., Some recent investigations concerning the sections of power series and related developments, Bull. Amer. Math. Soc. 42(1936) 505-522.

[147] Teodoriu, Luca, Sur les zéros de la dérivée d'une fonction holomorphe, Atti detta R. Accademia dei Lincei, No. 6, 13(1931) 591-593.

[148] Titus, C. J., A theory of normal curves and some applications, Pacific J. Math. 10(1960) 1083-1096.

[149] Titus, C. J., The combinatorial topology of an analytic function on the boundary of the disc, Acta Math. 106(1961) 45-64.

[150] Umezawa, T., On the theory of univalent functions, Tôhoko Math. J. 7(1955) 212-218.

[151] Van Vleck, E. B., On the location of roots of polynomials and entire functions, Bull. Amer. Math. Soc. 35(1929) 643-683.

[152] Verhandlungen Des Ersten Internationalen Mathematiker-Kongresses, ed. F. Rudio, Druck und Verlag von B. G. Teubner, Leipzig, 1898.

[153] Warschawski, S., On the higher derivatives at the boundary in conformal mapping, Trans. Amer. Math. Soc. 38(1935) 310- 340.

[154] Watson, G. N., Complex Integration and Cauchy's Theorem, Hafner Publishing Co., New York, 1914.

[155] Watson, G. N., A problem of analysis situs, Proc. London Math. Soc., Ser. 2, 15(1916) 227242 .

[156] Watson, G. N., Whittaker, E. T., A Course of Modern Analysis, $4^{\text {th }}$ edition, Cambridge University Press, 1935.

[157] Whitney, H., Moscow 1935: Topology Moving Toward America, "A Century of Mathematics in America", ed. P. L. Duren, Amer. Math. Soc., Providence, R. I., 1989, pp. 103-111.

[158] Wolff, J., L'intégral d'une fonction holomorphe et à partie réele positive dans une demi-plan est univalente, C. R. Acad. Sci. (Paris) 198(1934) 1209-1210.

[159] Young, W. H., On the Fourier series of bounded functions, Proc. London Math. Soc. 12(1913) 41-70.

[160] Zalcman, L., Real proofs of complex theorems, Amer. Math. Monthly 81(1974) 115-137.

Department of Mathematical Sciences, Villanova University, Villanova, PA 19085

E-mail address: frederick.hartmann@villanova.edu, alan.gluchoff@villanova.edu 